\title{
On fundamental groups related to degeneratable surfaces: conjectures and examples
}

\author{
Michael Friedman AND Mina TEICHER
}

\begin{abstract}
We argue that for a smooth surface $S$, considered as a ramified cover over $\mathbb{C P}^{2}$, branched over a nodal-cuspidal curve $B \subset \mathbb{C P}^{2}$, one could use the structure of the fundamental group of the complement of the branch curve $\pi_{1}\left(\mathbb{C P}^{2}-B\right)$ to understand other properties of the surface and its degeneration and vice-versa. In this paper, we look at embedded-degeneratable surfaces - a class of surfaces admitting a planar degeneration with a few combinatorial conditions imposed on its degeneration. We close a conjecture of Teicher on the virtual solvability of $\pi_{1}\left(\mathbb{C P}^{2}-B\right)$ for these surfaces and present two new conjectures on the structure of this group, regarding non-embedded-degeneratable surfaces. We prove two theorems supporting our conjectures, and show that for $\mathbb{C P}^{1} \times C_{g}$, where $C_{g}$ is a curve of genus $g, \pi_{1}\left(\mathbb{C P}^{2}-B\right)$ is a quotient of an Artin group associated to the degeneration.
\end{abstract}

Mathematics Subject Classification (2010): 14D06 (primary); 14Q10, 14H20, 14H30, 20F36 (secondary).

\section{Introduction}

Given a smooth algebraic projective variety $X$, one of the main techniques used to obtain information on $X$ is to degenerate it to a union of "simpler" varieties. The "simplest" degeneration can be thought as the degeneration of $X$ to a union of $\operatorname{dim} X$-planes, and one would like to use the combinatorial data induced from this arrangement of planes in order to find (or bound) certain invariants of $X$.

When $\operatorname{dim}(X)=1$, one would like to degenerate the curve into a line arrangement with only nodes as singularities. This has been thoroughly investigated. For example, it is known that any smooth plane curve can be degenerated into a union of lines. However, the situation for a curve in $\mathbb{C P}^{n}, n>2$ is completely different as there are, for example, smooth curves in $\mathbb{C P}^{3}$ which cannot be degenerated into a line arrangement with only double points (see [15]).

This work is partially supported by the Emmy Noether Research Institute for Mathematics (center of the Minerva Foundation of Germany).

Received February 14, 2010; accepted in revised form March 3, 2011. 
When $\operatorname{dim} X=2$, the problem of investigating projective surfaces in terms of their degeneration to a union of planes has only been investigated partially (see, for example, Zappa's papers from the 1940's [36] and [11] for a survey on this topic; see also [19] for degeneration of surfaces in $\left.\mathbb{C P}^{3}\right)$. Also, one should allow the existence of more complicated singularities in order to obtain degenerations. But there is another method to extract information on the surface, which is to consider it as a branched cover of the projective plane $\mathbb{C P}^{2}$ with respect to a generic projection. The motivation for this point of view is Chisini's conjecture (recently proved by Kulikov $[17,18]$ ): Let $B$ be the branch curve of generic projection $\pi: S \rightarrow \mathbb{C P}^{2}$ of degree at least 5 . Then $(S, \pi)$ is uniquely determined by the pair $\left(\mathbb{C P}^{2}, B\right)$. Moreover, if two surfaces $S_{1}$ and $S_{2}$ are deformation equivalent, then their branch curves $B_{1}$ and $B_{2}$ are isotopic. Thus, if the fundamental group $\pi_{1}\left(\mathbb{C}^{2}-B_{1}\right)$ is not isomorphic to $\pi_{1}\left(\mathbb{C}^{2}-B_{2}\right)$ then the surfaces are not deformation equivalent. This gives another motivation for considering $S$ in terms of its branch curve.

Therefore, it is reasonable to combine the two methods outlined above, i.e., investigating a projective surface $S$ and its degeneration $S_{0}$ by looking at their branch curves $B$ and $B_{0}$. Explicitly, we want to find the relations between the combinatorics of the planar degeneration and the fundamental group $\pi_{1}\left(\mathbb{C}^{2}-B\right)$.

Several works were done in this direction: for different embeddings of $\mathbb{C P}^{1} \times$ $\mathbb{C P}^{1}$, for the Veronese surface $V_{n}\left([29,30]\right.$ for $V_{n}, n \geq 3$ and [37] for $\left.V_{2}\right)$, for the Hirzebruch surfaces $F_{1,(a, b)}$ and $F_{2,(2,2)}([6,14])$, for $K 3$ surfaces $([13])$, for a few toric surfaces and for $\mathbb{C P}^{1} \times \mathbb{T}$ (where $\mathbb{T}$ is a complex torus, see [7]). For each surface in this list one can associate a graph $T$ to the degenerated surface. In all of the examples mentioned above the fundamental group $\pi_{1}\left(\mathbb{C}^{2}-B\right)$ is either a quotient of an associated Artin group $A(T)$ (except the Veronese surface $V_{2} \subset \mathbb{C P}^{5}$ ) or a quotient of a subgroup of $\widetilde{A}(T) \times \widetilde{A}(T)$ (where $\widetilde{A}(T)$ is a quotient of $A(T)$ by a single relation. For example, when $T$ is a tree with maximum valence 3 , then $A(T)$ is isomorphic to the braid group $B_{n}$, where $n=$ degree of the surface). In particular, once the embedding of the surface in a projective space is "ample enough", the structure of $\pi_{1}\left(\mathbb{C}^{2}-B\right)$ is of the mentioned second type. Thus, a natural question rises: what are the sufficient conditions on the degeneration such that $\pi_{1}\left(\mathbb{C}^{2}-\right.$ $B$ ) will be isomorphic to a quotient of a subgroup of $\widetilde{A}(T) \times \widetilde{A}(T)$ ? One of the goals of this paper is to give the conditions under which the fundamental group has this desired structure. These conditions are in a form of a local-global condition: if there are enough singular points in the degenerated surface satisfying a certain local condition, then the fundamental group is isomorphic to the quotient. Under these conditions, the conjecture posed in [34] regarding the virtual-solvability of the above fundamental group is proven.

Another main result deals with a new set of examples, not satisfying these conditions. The surfaces $\mathbb{C P}^{1} \times C_{g}$, where $C_{g}$ is a curve of genus $g \geq 1$, are studied, and for $g \geq 1$ the above fundamental group is computed. These new examples are essential for the second goal of this paper: to understand better these groups for non-simply connected surfaces. 
The structure of the paper is as follows. Section 2 examines the structure of the fundamental group. Subsections 2.1 and 2.2 introduce the main definitions and restrictions on the degeneration. We then state one of the main theorems in Subsection 2.3: that under a certain condition, there is an epimorphism from $\widetilde{B}_{n}^{(2)}$ to $\pi_{1}\left(\mathbb{C}^{2}-B\right)$. We also present two conjectures on the structure of $\pi_{1}\left(\mathbb{C}^{2}-B\right)$ when the condition does not hold (see Conjectures 2.25 and 2.26). In Subsection 2.4 we prove the main theorem from Subsection 2.3. In Section 3 we prove another main theorem, where we compute the group $G_{g}=\pi_{1}\left(\mathbb{C}^{2}-B_{g}\right)$, where $B_{g}$ is the branch curve of $\mathbb{C P}^{1} \times C_{g}$. We show that $G_{g}$ is (again) a quotient of $A(T)$, and also compute $\pi_{1}\left(\left(\mathbb{C P}^{1} \times C_{g}\right)_{\text {Gal }}\right)$ - the fundamental group of the Galois cover of $\mathbb{C P}^{1} \times C_{g}$.

ACKnowledgements. We thank Alberto Calabri and Ciro Ciliberto for referring the first author to their paper [9] and for fruitful discussions during the "School (and Workshop) on the Geometry of Special Varieties" which was held in 2007 at the IRST, Fondazione Bruno Kessler in Povo (Trento). We also would like to thank Christian Liedtke and Robert Schwartz for stimulating talks and important discussions.

\section{Degenerations and fundamental groups}

In this section we examine the structure of the fundamental group of the complement of the branch curve, under some assumptions. Subsection 2.1 introduces the main definitions and notation. We state the main theorem on the structure of the fundamental group of the complement of the branch curve in $\mathbb{C}^{2}$, under certain conditions, in Subsection 2.3 and also present two conjectures on the structure of the fundamental group regarding surfaces which do not satisfy the desired conditions. The virtual-solvability of this fundamental group is discussed in Subsection 2.3.1, together with the class of surfaces satisfying the desired conditions. In Subsection 2.4 we prove the main theorem.

\subsection{Notation for planar degeneration}

We begin with a few definitions.

\section{Definition 2.1.}

(i) Degeneration: Let $\Delta$ be the complex unit disc. A degeneration of surfaces, parametrized by $\Delta$ is a proper and flat morphism $\rho: S \rightarrow \Delta$ (where $S$ is a 3-dim variety) such that each fibre $S_{t}=\rho^{-1}(t), t \neq 0$ (where 0 is the closed point of $\Delta$ ), is a smooth, irreducible, projective surface. The fiber $S_{0}$ is called the central fiber. A degeneration $\rho: S \rightarrow \Delta$ is said to be embedded in $\mathbb{C P}^{r}$ if there is an inclusion $i: S \hookrightarrow \Delta \times \mathbb{C P}^{r}$ and, when denoting the projection $p_{1}: \Delta \times \mathbb{C P}^{r} \rightarrow \Delta$, then $p_{1} i=\rho$. 
(ii) Planar degeneration: When the central fiber $S_{0}$ in the above embedded degeneration is a union of planes, then we call the degeneration a planar degeneration. A survey on degeneratable surfaces can be found in [11]. Examples of planar degenerations can be found in [9] (for scrolls), [23] (for Hirzebruch surfaces), [28] (for veronese surfaces), [21] (for $\mathbb{C P}^{1} \times \mathbb{C P}^{1}$ ).

(iii) Regeneration: The regeneration methods are actually, locally, the reverse process of the degeneration method. In this article it is used as a generic name for finding a degeneration $\rho: S \rightarrow \Delta$ when the central fiber $S_{0}$ is given. In fact, one can deduce what is the effect of a regeneration on the corresponding branch curves. The regeneration rules (see Subsection 3.1.2) explain the effect of the regeneration on the braid monodromy factorization (see Subsection 3.1) of the branch curves of the fibers.

(iv) Local fundamental group: Given a planar degeneration $\rho: S \rightarrow \Delta$, denote by $B_{t}$ the branch curve of a generic projection of $S_{t}$ to $\mathbb{C P}^{2}$ (such that the center of projection is the same for every $t$ ). Given a singular point $p \in B_{0}$ choose a small neighborhood $U$ of $p$ such that $U \cap \operatorname{Sing}\left(B_{0}\right)=\{p\}$. Since $S_{0}$ is a planar degeneration, there are lines $\ell_{i}$ such that $U \cap B_{0}=\cup\left(U \cap \ell_{i}\right)$, such that $\cap \ell_{i}=\{p\}$. Assume that for the branch curve $B_{t}$ of general fiber $S_{t}, t \neq 0$, we have that $\lim _{t \rightarrow 0}\left(U \cap \operatorname{Sing}\left(B_{t}\right)\right)=\{p\}$. The local fundamental group of $p$ is defined as $\pi_{1}\left(U-B_{t}\right)$ and we denote it by $G_{p}$.

Let $S_{1} \subset \mathbb{C P}^{N}$ be a smooth surface of degree $n$ which admits a planar degeneration $\rho: S \rightarrow \Delta$, and let $f: \mathbb{C P}^{N} \rightarrow \mathbb{C P}^{2}$ be the generic projection with respect to $S_{t}$, for every $t$. We denote by $R$ the ramification curve of $S_{1}$ and by $B \subset \mathbb{C P}^{2}$ its branch curve with respect to a generic projection $\left.\pi \doteq f\right|_{S_{1}}: S_{1} \rightarrow \mathbb{C P}^{2}$. Also, let $G \doteq \pi_{1}\left(\mathbb{C P}^{2}-B\right)$.

We denote by $S_{0}$ the planar degeneration of $S_{1}$ (the central fiber of $\rho$ ), i.e. $S_{0}$ is a union of planes. Let

$$
S_{0}=\bigcup_{i=1}^{n} \Pi_{i}
$$

such that each $\Pi_{i}$ is a plane.

Notation 2.2. $n=\operatorname{deg} S_{0}=\operatorname{deg} S_{1}$.

Let $\left.\pi_{0} \doteq f\right|_{S_{0}}: S_{0} \rightarrow \mathbb{C P}^{2}$ be the generic projection of $S_{0}$ to $\mathbb{C P}^{2}$. In this case, the ramification curve also degenerates into a union of $\ell$ lines

$$
R_{0}=\bigcup_{i=1}^{\ell} L_{i}
$$

and thus the degenerated branch curve is of the form

$$
\pi_{0}\left(R_{0}\right)=B_{0}=\bigcup_{i=1}^{\ell} l_{i},
$$

where $l_{i}=\pi_{0}\left(L_{i}\right)$. 
Notation 2.3. $\ell=\operatorname{deg} R_{0}=\operatorname{deg} B_{0}$.

Since $R_{0}$ is an arrangement of lines in $\mathbb{C P}^{N}$, these lines can intersect each other.

Notation 2.4. $m^{\prime}=$ the number of points $\left\{P_{i}\right\}_{i=1}^{m^{\prime}}$ which lie on more than one line $L_{j}$.

For a point $x \in L_{i}$ (or $x \in l_{i}$ ) let $v(x)$ be the number of distinct lines in $R_{0}$ (or $B_{0}$ ) on which $x$ lies. For example, if $x \in\left\{P_{i}\right\}_{i=1}^{m^{\prime}}$, then $v(x)>1$.

Notation 2.5. Denote by $P=\left\{x \in B_{0}: v(x)>1\right\}$, and let $p_{i}=\pi_{0}\left(P_{i}\right) \in B_{0}$ (note that $\left.v\left(p_{i}\right)=v\left(P_{i}\right)\right)$. Denote $P^{\prime} \doteq\left\{p_{i}\right\}_{i=1}^{m^{\prime}}$.

Remark 2.6. Note also that $P^{\prime}=\left\{p_{i}\right\}_{i=1}^{m^{\prime}} \varsubsetneqq P$, since there are points (called parasitic intersection points; see the explanation in Subsection 2.4.1) which are in $P$ but not in $\left\{p_{i}\right\}_{i=1}^{m^{\prime}}$.

Notation 2.7. Recall that $R_{0}=\cup L_{i}$. Define the set of lines

$$
M \doteq\left\{L_{i} \in R_{0}: \text { there is only one point } x \in L_{i} \text { such that } v(x)>1\right\} .
$$

For each $l \in M$, choose a point $y_{l} \in l$ s.t. $v\left(y_{l}\right)=1$. Denote

$$
Y \doteq\left\{y_{l}\right\}_{l \in M} ;
$$

the set of points is called the set of 2-point.

We recall the definition of $\widetilde{B}_{n}$, since the local fundamental group of many of the singular points of $B_{0}$ is strongly related to this group.

\section{Definition 2.8.}

(1) Let $X, Y$ be two half-twists in the braid group $B_{n}=B_{n}(D, K)$ (see Subsection 3.1 for the notation $D, K)$. We say that $X, Y$ are transversal if they are defined by two simple paths $\xi, \eta$ which intersect transversally in one point different from their ends.

(2) Let $N$ be the normal subgroup of $B_{n}$ generated by conjugates of [X, $\left.Y\right]$, where $X, Y$ is a transversal pair of half-twists. Define

$$
\widetilde{B}_{n}=B_{n} / N \text {. }
$$

Let $x_{1}, \ldots, x_{n-1}$ be the standard generators of $B_{n}$. Equivalently, we can define

$$
\widetilde{B}_{n}=B_{n} /\left\langle\left[x_{2}, x_{3}^{-1} x_{1}^{-1} x_{2} x_{1} x_{3}\right]\right\rangle
$$

for $n>3$. Recall that we can define on $B_{n}$ two natural homomorphism:

(i) $\operatorname{deg}: B_{n} \rightarrow \mathbb{Z}$ s.t. $\operatorname{deg}\left(\prod x_{i}^{n_{i}}\right)=\sum n_{i}$. 
(ii) $\sigma: B_{n} \rightarrow S_{n}$ s.t. $\sigma\left(x_{i}\right)=(i i+1)$. For properties of $\widetilde{B}_{n}$ see, for example, [22,31,35].

(3) The following group plays an important role in finding a presentation of a fundamental group of the complement of the branch curve. Define, as in [8], the group

$$
\widetilde{B}_{n}^{(2)} \doteq\left\{(x, y) \in \widetilde{B}_{n} \times \widetilde{B}_{n}, \operatorname{deg}(x)=\operatorname{deg}(y), \sigma(x)=\sigma(y)\right\} .
$$

Definition 2.9. Recall that for $p \in P^{\prime}=\left\{p_{i}\right\}_{i=1}^{m^{\prime}}$, we denote by $G_{p}$ the local fundamental group (see Definition 2.1(iv)). Define the following set:

$$
Q \doteq\left\{p \in\left\{p_{i}\right\}_{i=1}^{m^{\prime}}: \text { there exists an epimorphism of } \widetilde{B}_{v(p)}^{(2)} \rightarrow G_{p} \text {, and } v(p)>3\right\}
$$

and denote

$$
|Q|=m \text {. }
$$

Thus, we have the following relations between the sets of points:

$$
Q \doteq\left\{x_{j}\right\}_{j=1}^{m} \subset P^{\prime} \doteq\left\{p_{i}\right\}_{i=1}^{m^{\prime}} \subset P .
$$

Remark 2.10. The definition of $Q$ is not meaningless: there are singular points $p \in$ $\left\{p_{i}\right\}_{i=1}^{m^{\prime}}, v(p)>3$ which occur during the (described above) degeneration process and have an epimorphism $\widetilde{B}_{v(p)}^{(2)} \rightarrow G_{p}$, where $G_{p}$ is the local fundamental group associated to $p$. For example, let $p_{6}$ (respectively $p_{5}$ ) a singular point of $S_{0}$ called a 6-point (respectively 5-point) which is locally an intersection of 6 (respectively 5) planes at a point, whose regeneration is described at [22] [23, Definition 4.3.3] (respectively [12]). Then $G_{p_{i}}$ is isomorphic to a quotient of $\widetilde{B}_{i}^{(2)}$ for $i=6,5$. For the 4-point $p_{4}$ (s.t. its regeneration is described at [22,31]), we get that $G_{p_{4}} \simeq \widetilde{B}_{4}$, which is also a quotient of $\widetilde{B}_{4}^{(2)}$.

Definition 2.11 $\left(\mathrm{Graph}_{S_{0}}\right)$. We define the graph $\mathrm{Graph}_{S_{0}}$. The vertices are the $m^{\prime}$ points $\left\{P_{i}\right\}_{i=1}^{m^{\prime}}$ and the set $Y$ of 2-points. Two vertices in $\mathrm{Graph}_{S_{0}}$ are connected by an edge if both of the corresponding points on $R_{0}$ lie on a unique line $L_{i} \subset R_{0}$.

We want to defined boundary and interior (non-boundary) vertices of Verti$\operatorname{ces}\left(\mathrm{Graph}_{S_{0}}\right)$.

Definition 2.12. There are triples of edges $e_{i}, e_{j}, e_{k} \in \operatorname{Edges}\left(\operatorname{Graph}_{S_{0}}\right)$ such that their union is a triangle $T_{i j k}$. We define the following subset of the vertices of $\mathrm{Graph}_{S_{0}}$, called the boundary vertices.

$$
\begin{aligned}
& V_{B}=\left\{p \in \operatorname{Vertices}\left(\mathrm{Graph}_{S_{0}}\right): p\right. \text { is not a vertex } \\
& \text { of two (or more) different triangles } \left.T_{i j k}\right\} .
\end{aligned}
$$

Note that $Y \subset V_{B}$. Also, denote

$$
V_{B}^{c}=\operatorname{Vertices}\left(\operatorname{Graph}_{S_{0}}\right) \backslash V_{B} .
$$

The subset $V_{B}^{c}$ is called the interior vertices. 
Example 2.13. The interior and boundary points, for the degeneration of the Hirzebruch surface $F_{1,(2,2)}$ :

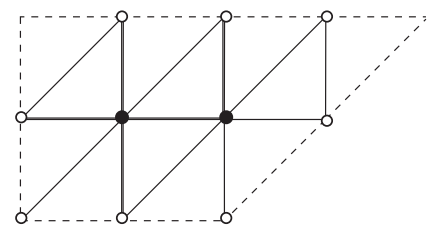

Figure 2.1. The white vertices are the boundary vertices $V_{B}$ and the black vertices are interior vertices $V_{B}^{c}$.

Remark 2.14. We have two inequalities which relate the above constants.

(1) Assume that the degree of the ramification curve of $S$ is $2 \ell$ (which will be one of the conditions imposed on $S$. see condition (3) in Definition 2.15). we have that $2 \ell \geq 2 n-2$ (which follows from the fact that $S$ is a ramified cover of $\mathbb{C P}^{2}$ ) or

$$
n \leq \ell+1
$$

(2) Denote by $\bar{m}$ the number of vertices in $\mathrm{Graph}_{S_{0}}$ (see Definition 2.11), by $\bar{\ell}$ the number of edges in $\mathrm{Graph}_{S_{0}}$ and $\bar{n}$ the number of triangles in Graph$S_{S_{0}}$. Note that $n>\bar{n}, \bar{m}>m$ and $\bar{\ell}=\ell$. By the Euler characteristic for planar graphs we get $\bar{m}-\bar{\ell}+\bar{n}=1$ or

$$
\bar{n}-1<\ell-m \text {. }
$$

\subsection{Conditions on the planar degeneration}

In this subsection, we introduce the following conditions that our projective surface $S$ has to satisfy.

Definition 2.15. A surface $S=S_{1}$ is called simply-degeneratable surface if it satisfies the following three conditions:

Condition (1) $S$ admits a planar degeneration, i.e., $\exists \rho: \tilde{S} \rightarrow \Delta$ s.t. $\rho^{-1}(1)=$ $S_{1}=S, \rho^{-1}(0)=S_{0}$ and $S_{0}$ is a union of planes.

Condition (2) The degeneration of $S$ to $S_{0}$ induces a degeneration of the branch curve $B$ to $B_{0}$ that satisfies the following condition: For a plane curve $C \subset \mathbb{C P}^{2}$, let $\operatorname{Sing}(C)$ be the singularities of $C$ with respect to a fixed generic projection $C \rightarrow$ $\mathbb{C P}^{1}$. Denote $\operatorname{Sing}_{0} \doteq \operatorname{Sing}\left(B_{0}\right), \operatorname{Sing}_{t}=\operatorname{Sing}\left(B_{t}\right), t \neq 0$. For each $p \in \operatorname{Sing}_{0}$ consider a small enough neighborhood $U_{p}$ of $p$ as in Definition 2.1(iv). We require that the set $\operatorname{Sing}(B) \backslash \bigcup_{p \in \operatorname{Sing}_{0}}\left(U_{p} \cap \operatorname{Sing}(B)\right)$ contains only simple branch points.

Condition (3) The degeneration of the branch curves $B \rightarrow B_{0}$ is two-to-one (see $[29,31]$ for further details on two-to-one degenerations of branch curve), that is $\operatorname{deg} B=2 \operatorname{deg} B_{0}$. 
Remark 2.16. We show that the three conditions above are independent. As we are interested in planar degenerations, we look at the following examples when the degeneration already satisfies Condition (1).

(1) A degeneration of a smooth cubic surface in $\mathbb{C P}^{3}$ (whose branch curve is a sextic with six cusps) into a union of three planes, all of them intersecting in a line, is an example of a surface which does not satisfy Conditions (2), (3).

(2) A degeneration of a union of three generic hyperplanes in $\mathbb{C P}^{3}$ (whose branch curve is a union of three lines, intersecting at three different points) into a union of three hyperplanes meeting at a single point, is an example of a degeneration that satisfies Condition (2) but not (3).

(3) A degeneration of a cone over a smooth conic in $\mathbb{C P}^{2}$ into a union of two hyperplanes is an example of a degeneration that satisfies Condition (3) but not (2).

(4) An example of planar degeneration that satisfies Conditions (2), (3) is a degeneration of a smooth quadric in $\mathbb{C P}^{3}$ into a union of two hyperplanes.

We now define a fourth condition imposed on the degeneration: that every boundary vertex has at least one interior vertex as a "neighbor" (see Definition 2.12).

Definition 2.17. A surface $S$ is called embedded-degeneratable surface if it is a simply-degeneratable surface and it satisfies the following fourth condition:

Condition (4) We require that for each boundary vertex $p \in V_{B}$ there exist an interior vertex $p^{c} \in V_{B}^{c}$ and an edge $e_{p} \in \operatorname{Edges}\left(\operatorname{Graph}_{S_{0}}\right)$ s.t. $e_{p}$ connects $p$ and $p^{c}$.

Example 2.18. The fourth condition is imposed in order to avoid degenerations as depicted in the following picture. Figure 2.2[1] presents a degeneration with no interior points $\left(V_{B}^{c}=\emptyset\right)$. Figure 2.2[2] presents a degeneration with not enough neighboring interior vertices (though $V_{B}^{c} \neq \varnothing$ ). By definition, the dashed border lines are not a part of Edges $\left(\operatorname{Graph}_{S_{0}}\right)$.

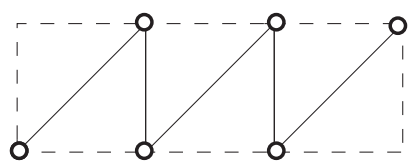

$[1]$

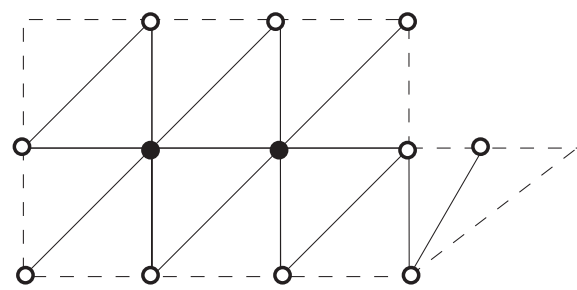

[2]

Figure 2.2. Degenerations which do no satisfy Condition (4). The white vertices are the boundary vertices $V_{B}$ and the black vertices are interior vertices $V_{B}^{c}$.

The following degeneration is a degeneration that satisfies all the four conditions. 


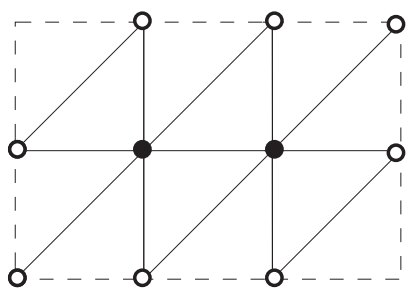

Figure 2.3. Allowable degeneration of $\mathbb{C P}^{1} \times \mathbb{C P}^{1}$. The white vertices are the boundary vertices $V_{B}$ and the black vertices are interior vertices $V_{B}^{c}$.

Remark 2.19. Assume that there are interior vetrices in a given degeneration $\left(V_{B}^{c} \neq \emptyset\right)$. Then in the case of a toric degeneration any degeneration always satisfies Condition (4). However, this is not known for general degenerations.

\subsection{Necessary condition on $\pi_{1}\left(\mathbb{C}^{2}-B\right)$}

We present here the main result for this section - under which conditions is $\pi_{1}\left(\mathbb{C}^{2}-\right.$ $B$ ) a quotient of $\widetilde{B}_{n}^{(2)}$. We begin with two examples:

Example 2.20. For the degeneration of the Hirzebruch surface $F_{1,(2,2)}$ :

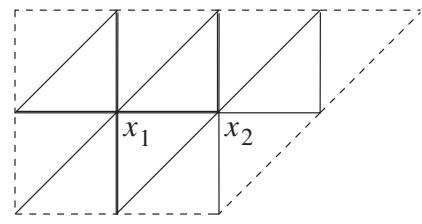

Figure 2.4. The degeneration of $F_{1,(2,2)}$. Note that the dashed border lines are not a part of the ramification curve

we have $Q=\left\{x_{1}, x_{2}\right\}$ (see [14] for the calculation of the local fundamental groups) and $m=2, \ell=13, n=12$, as depicted above. Note that in this case $\ell-m \leq n-1$.

Example 2.21. For the degeneration of the surface $\mathbb{C P}^{1} \times \mathbb{T}$ (where $\mathbb{T}$ is a torus), embedded with respect to the linear system $(2,3)$

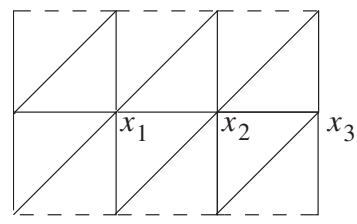

Figure 2.5. The degeneration of $\left(\mathbb{C P}^{1} \times \mathbb{T}\right)_{(2,3)}$. Note that the dashed horizontal border lines are not a part of the ramification curve and the vertical are. The vertical border lines are identified.

We have $m=3, \ell=15, n=12$ (as $\left.Q=\left\{x_{1}, x_{2}, x_{3}\right\}\right)$, and the inequality $\ell-m \leq$ $n-1$ is not satisfied. 
These observations lead us to state the following theorem.

Theorem 2.22. Let $S$ be a smooth embedded-degeneratable projective surface. Let $B \subset \mathbb{C}^{2}$ its branch curve with respect to a generic projection, $B_{0}$ its degeneration. Denote $\ell=\frac{1}{2} \operatorname{deg} B, n=\operatorname{deg} S, m=$ number of singular points $p$ of $B_{0}$ whose local fundamental group is a quotient of $\widetilde{B}_{v(p)}^{(2)}$ (see Definitions 2.1, 2.9).

If $\ell-m \leq n-1$ then there exist an epimorphism $\widetilde{B}_{n}^{(2)} \rightarrow G=\pi_{1}\left(\mathbb{C}^{2}-B\right)$.

The proof of this theorem will be given in Subsection 2.4.

Example 2.23. We give here a list of known surfaces, satisfying Theorem 2.22: $\mathbb{C P}^{1} \times \mathbb{C P}^{1}$ embedded with respect to the linear system $a l_{1}+b l_{2}$, where $a, b>1$ (see [22]), the Veronese surface $V_{n}, n \geq 3$ (see [29,30]), the Hirzebruch surfaces $F_{1}$ (embedded with respect to the linear system $a C+b E_{0}$, where $a, b>1, C, E_{0}$ generate the Picard group of $F_{1}$, see [14]) and $F_{2}$ (embedded with respect to the linear system $2 C+2 E_{0}$ (see [6]), and a few families of $K 3$ surfaces (see [13]).

Before proving the theorem, we want to review a few surfaces for which the condition in Theorem 2.22 does not hold, presenting two conjectures.

For the first conjecture we need the following definition.

Definition 2.24. (1) Given an Artin group $A$, generated by $\left\{x_{i}\right\}_{i=1}^{r}, r>2$, we define the following quotient:

$$
\widetilde{A}=A /\left\langle\left[x_{2}, x_{3}^{-1} x_{1}^{-1} x_{2} x_{1} x_{3}\right]\right\rangle
$$

(2) Let deg be the following epimorphism: $\operatorname{deg}: A \rightarrow \mathbb{Z}$ s.t. $\operatorname{deg}\left(\prod x_{i}^{n_{i}}\right)=\sum n_{i}$. Assume there exists an epimorphism from $A$ to the symmetric group $\sigma: A \rightarrow S_{n}$. In this case, define

$$
\widetilde{A}^{(2)} \doteq\{(x, y) \in \widetilde{A} \times \widetilde{A}, \operatorname{deg}(x)=\operatorname{deg}(y), \sigma(x)=\sigma(y)\} .
$$

The first conjecture on the structure of $G=\pi_{1}\left(\mathbb{C}^{2}-B\right)$ is similar to Theorem 2.22 , when $Q \neq \emptyset$ but does not contain enough points.

Conjecture 2.25. For a smooth embedded-degeneratable surface $S$ s.t. $|Q|=m \geq$ 1 and $\ell-m>n-1$ (i.e. does not satisfy the condition in Theorem 2.22) one can associate a graph $T$ and an Artin group $A(T)$ such that $G$ is a quotient of $\widetilde{A(T)}{ }^{(2)}$.

The condition above means that $S$ has a planar degeneration with 2:1 degeneration of the branch curve, whose degeneration has singular points in the set $Q$, but not enough. For example, See [7, Conjecture 3.7] (on the embedding of $\mathbb{C P}^{1} \times \mathbb{T}$ with respect to the linear system $(m, n), m, n>1)$ and [3] (for the degeneration of $\mathbb{T} \times \mathbb{T})$.

We now review a few surfaces for which the set $Q$ is empty. 
Conjecture 2.26. For a simply-degeneratable surface $S$ such that the set $Q=\varnothing$ (i.e. the degeneration has only boundary points (see Definition 2.12)) and such that $G=\pi_{1}\left(\mathbb{C}^{2}-B\right)$ has "enough" commutation relations (see Remark 2.27), we conjecture that one can associate a graph $T$ and an Artin group $A(T)$ such that $G$ is a quotient of $A(T)$.

Remark 2.27. Recall that $G$ has the natural monodromy epimorphism $\varphi: G \rightarrow S_{n}$ $(n=\operatorname{deg}(S)$ ), defined by sending each generator to a transposition, describing the sheets which are exchanged. By "enough" commutation relations we mean that for $a, b \in G$ such that $\varphi(a), \varphi(b)$ are disjoint transpositions, then $a, b$ commute.

Example 2.28. (1) The surface $\mathbb{C P}^{1} \times \mathbb{C P}^{1}$ (embedded with respect to the linear system $l_{1}+b l_{2}, b \geq 1$ and denoted as $\left.\left(\mathbb{C P}^{1} \times \mathbb{C P}^{1}\right)_{(1, b)}\right)$ and the Hirzebruch surface $F_{1}$ (embedded with respect to the linear system $a C+E_{0}, a \geq 1$ and denoted as $\left.F_{1,(1, a)}\right)$ were investigated in [5]. They do not satisfy condition (4) (see Definition 2.17 ) and also the main condition in Theorem 2.22. In both of these cases, however, the fundamental group $\pi_{1}\left(\mathbb{C}^{2}-B\right)$ is a quotient of the braid group $B_{n}$, or equivalently a quotient of the Artin group $A(T)$, where $T$ is depicted in the following figure.

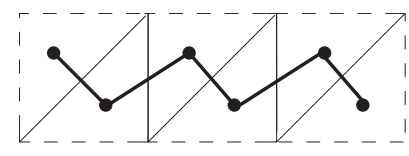

[1]

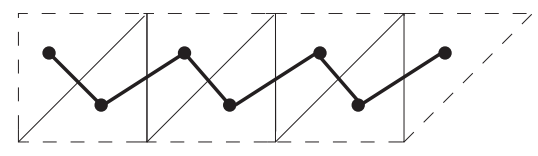

[2]

Figure 2.6. The degeneration of $\left(\mathbb{C P}^{1} \times \mathbb{C P}^{1}\right)_{(1,3)}$ (Figure [1]) and $F_{1,(1,3)}$ (Figure [2]) and their associated graphs $T$.

(2) The Veronese surface $S=V_{2} \subset \mathbb{C P}^{5}$ and its associated fundamental group $\pi_{1}\left(\mathbb{C}^{2}-B_{S}\right)$ were investigated in $[24,37]$. Also in this example $V_{2}$ and its degeneration do not satisfy the necessary conditions. Note that this is an exceptional case to the previous example, as $\pi_{1}\left(\mathbb{C}^{2}-B_{S}\right)$ is not isomorphic to a quotient of $A(T)$, where $T$ is depicted in the following figure.

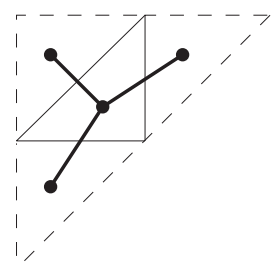

Figure 2.7. The degeneration of $V_{2}$ and its associated graph $T$.

This can be seen from [37], as $\pi_{1}\left(\mathbb{C P}^{2}-B_{S}\right)$ is generated by four generators. The fact that $G=\pi_{1}\left(\mathbb{C}^{2}-B_{S}\right)$ does not have commutation relations is the reason we 
require that "enough" commutation relations in Conjecture 2.26 will hold (indeed, the condition in Remark 2.27 is not satisfied with respect to the map $G \rightarrow S_{4}$ ).

Note that the Veronese surface $V_{2}$ is exceptional also for other statements in classical algebraic geometry - it is, for example, the only counter example to the Chisini's conjecture [18].

\subsubsection{Virtual solvability of $G$}

For surfaces whose planar degeneration satisfy the condition introduced in Theorem 2.22, the conjecture on the structure (and the virtual solvability) of $G$ proposed in [34] is correct. This is due to the fact that by [8, Remarks 3.7, 3.8], if there is an epimorphism $\widetilde{B}_{n}^{(2)} \rightarrow G$, then $G$ is virtually solvable. However, these conditions imply that the class of embedded-degeneratable surfaces is rather small; for example, if $\pi_{1}(S)$ contains a free group of rank 2 , then $G$ is not virtually solvable (see [20]). These type of surfaces is the main topic of Section 3.

Also, by [20, Corollary 4.9, Proposition 4.11] one can compute explicitly $\operatorname{rank}\left(H_{1}\left(X_{\mathrm{Gal}}, \mathbb{Z}\right)\right)$ (where $X_{\mathrm{Gal}}$ is the Galois cover of $X$. see subsection 3.4$)$, and if $X$ is simply connected, one can also find $\pi_{1}\left(X_{\mathrm{Gal}}\right)$.

\subsection{Proof of the main theorem}

We first cite the theorem we want to prove (Theorem 2.22):

Let $S$ be a smooth embedded-degeneratable projective surface. Let $B \subset \mathbb{C}^{2}$ its branch curve with respect to a generic projection, $B_{0}$ its degeneration. Denote $\ell=\frac{1}{2} \operatorname{deg} B, n=\operatorname{deg} S, m=$ number of singular points $p$ of $B_{0}$ whose local fundamental group is a quotient of $\widetilde{B}_{v(p)}^{(2)}$ (see Definitions 2.1, 2.9).

$$
\text { If } \ell-m \leq n-1 \text { then there exist an epimorphism } \widetilde{B}_{n}^{(2)} \rightarrow G=\pi_{1}\left(\mathbb{C}^{2}-B\right) .
$$

Proof. We introduce the following notation.

Notation 2.29. Let $S_{0}=\cup^{n} \Pi_{i}$ be the degeneration of $S$ as above, $R_{0}$ the degenerated ramification curve. We build the graph $S_{0}^{*}=(E, V)$ called the dual graph to $S_{0}$ by the following procedure (see also [23, page 532]). each plane $\Pi_{i}$ corresponds to a vertex $v_{i} \in V, 1 \leq i \leq n$, and each line $\Pi_{k} \cap \Pi_{j}=L_{i} \in R_{0}$ corresponds to an edge $e_{i} \in E, 1 \leq i \leq \ell$, connecting the vertices $v_{k}$ and $v_{j}$. For example

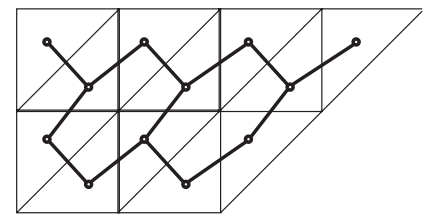

Figure 2.8. The dual graph $S_{0}^{*}$ of the degeneration of $F_{1,(2,2)}$.

We first prove the following lemma: 
Lemma 2.30. There exists a spanning subtree of $S_{0}^{*}$ with $\ell-m$ edges if and only if $\ell-m \leq n-1$.

Proof. If $n$ is the number of vertices in a connected graph, then if the number of edges is greater than $n-1$, then there are cycles in the graph. Therefore, if there is a spanning connected subtree of $S_{0}^{*}$ with $\ell-m$ edges, then $\ell-m \leq n-1$.

For the other direction, assume first that $\ell-m=n-1$. For $x \in\left\{p_{i}\right\}_{i=1}^{m^{\prime}}$ we denote by $L_{x}$ the set of lines such that $x$ lies on them, and let $L_{x}^{*}$ be the set of edges in $S_{0}^{*}$ corresponding to $L_{x}$. We create a new graph $T_{0}^{*}=\left(E_{T}, V_{T}\right)$ from $S_{0}^{*}$. The vertices of $T_{0}^{*}$ will be the same as $S_{0}^{*}$, but for each $x \in Q$ we erase one edge $e_{x}$ from $S_{0}^{*}$, such that $e_{x} \in L_{x}^{*}$. Since for each $x \in Q, v(x)>3$, we demand that if there exist $x, y \in Q$ such that $x$ and $y$ are neighbors (i.e. there exist a line $L$ s.t. $x, y \in L$ ), then $e_{x} \cap e_{y}=\varnothing$. We choose the $e_{x}$ 's satisfying the above requirements. Let us note that $m$ can be equal to 1 , so the choice of $y$ above is irrelavant. We now show that the resulting graph $T_{0}^{*}$ is connected.

Note that if $x, y$ are neighbors, then locally the graphs $S_{0}$ and $S_{0}^{*}$ would look as in the following figure:

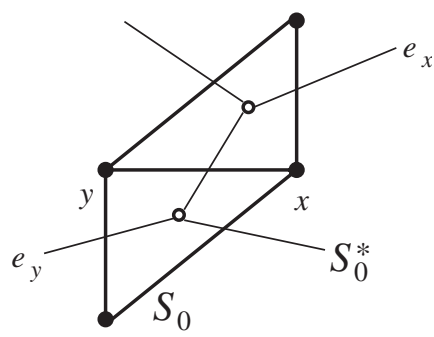

Figure 2.9. Local neighborhood of two vertices.

since the degeneration is planar. Thus we can choose $e_{x}$ and $e_{y}$ as depicted in Figure 2.7 and the resulting graph will be connected. Now one can proceed by induction to prove connectedness. Note that the number of edges in $T_{0}^{*}$ is $\ell-m$. Since $\ell-m=n-1, T_{0}^{*}$ is a spanning subtree of $S_{0}^{*}$, by definition.

If $\ell-m<n-1$ there exist $k \in \mathbb{N}, k<m$ such that $\ell-k=n-1$. We now choose $k$ points from $Q$, and proceed as before to construct $T_{0}^{*}$.

For example, the following figure presents a possible spanning subtree $T_{0}^{*}$ for the degeneration of $F_{1,(2,2)}$ :

Figure 2.10.

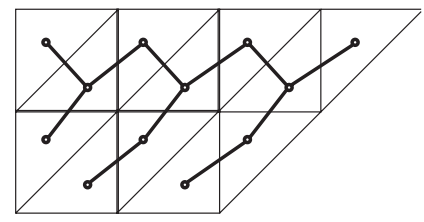


By Lemma 2.30, there exists a spanning subtree $T_{0}^{*}$. We refine the construction of $T_{0}^{*}$ in the following way. By our assumptions, for each $x \in Q$, there exists an epimorphism $\widetilde{B}_{v(x)}^{(2)} \rightarrow G_{x}$, where $G_{x}$ is the local fundamental group of $x$. As can be seen from Definition 2.8, $\widetilde{B}_{v(x)}^{(2)}$ is generated by pairs $\left\{\Gamma_{i}, \Gamma_{i}^{\prime}\right\}_{i=1}^{v(x)-1}$, when the $\Gamma_{i}$ 's are the standard generators of $\widetilde{B}_{v(x)}$. However, by the Van Kampen Theorem (see Theorem 3.6), using the fact that the degeneration reduces the degree of the branch curve by half (by Condition (3) on $S$. See Definition 2.15), we see that $G_{x}$ is generated by pairs of (topological) generators $\left\{\gamma_{i}, \gamma_{i^{\prime}}\right\}_{i=1}^{v(x)}$. Thus, we can choose to express two generators $\gamma, \gamma^{\prime} \in\left\{\gamma_{i}, \gamma_{i^{\prime}}\right\}_{i=1}^{v(x)}$ by the other generators s.t. the pair $\gamma, \gamma^{\prime}$ corresponds to a degenerated line $L \in L_{x}$ and its corresponding edge $e_{x} \in L_{x}^{*}$ will be the edge which we erase (possibly after renumeration of the generators of $\widetilde{B}_{v(x)}^{(2)}$ such that the erased edge will satisfy the demands imposed on it as in Lemma 2.30) in order to get $T_{0}^{*}$.

Remark 2.31. Note that for all $x \in\left\{p_{i}\right\}_{i=1}^{m^{\prime}}$ we erase at most one edge from $L_{x}^{*}$.

It is clear that for each $x \in Q$ there exists an embedding $\widetilde{B}_{v(x)}^{(2)} \hookrightarrow G$. Therefore $G_{x} \simeq \widetilde{B}_{v(x)}^{(2)} / R_{v(x)} \hookrightarrow G$ where $R_{v(x)}=\operatorname{ker}\left(\widetilde{B}_{v(x)}^{(2)} \rightarrow G_{x}\right)$.

Remark 2.32. The embedding $\widetilde{B}_{v(x)}^{(2)} \hookrightarrow G$ might be possible only after a conjugation of the generators $\Gamma_{i}, \Gamma_{i^{\prime}}$ by a certain power of $\sigma_{i}$ (which is a generator of the braid group). See, for example, [8, Subsection 6.1.2].

Let us now look at the points $x \in P \cup Y, x \notin Q$ : these are the points whose corresponding local fundamental group is not $\widetilde{B}_{v(x)}^{(2)}$. We start, in the following subsection, with the most important case, and later we remark on two more cases.

\subsubsection{Parasitic intersection points}

Each point $x \in B_{0}$ such that $v(x)=2$ is an intersection of two lines $l_{i}, l_{j}$. This kind of point, when $x \in P, x \notin\left\{p_{i}\right\}_{i=1}^{m^{\prime}}$ is called a parasitic intersection point. These points are not a projection of singular points of $R_{0}$, hence we get them as a result of the projection to $\mathbb{C P}^{2}$. During the regeneration process (see Subsection 3.1.2), each line is doubled, so eventually we get 4 nodes in $R$, and thus the local fundamental group is $\left\{\Gamma_{i}, \Gamma_{i^{\prime}}, \Gamma_{j}, \Gamma_{j^{\prime}}:\left[\Gamma_{\underline{i}},\left(\Gamma_{j}\right)_{\alpha}\right]=1\right\}$, where $\Gamma_{\underline{i}}=\Gamma_{i}$ or $\Gamma_{i^{\prime}}$ and $\alpha \in B_{n}$. Examining these relations together, it can be seen easily that $\alpha$ can be written as a product of generators which commute with $\Gamma_{i}$ (see [26, Theorem IX.2.2], since this arrangement of lines is a partial arrangement to what is called in [26, section IX, §1] dual to generic). Therefore, from the parasitic intersection points we induce the commutator relations between different generators $\Gamma_{\underline{i}}, \Gamma_{\underline{j}}$ such that the corresponding lines $L_{i}, L_{j}$ do not have a vertex in common.

Notation 2.33. Denote the set of all relations induced from the parasitic intersection points as $R_{\mathrm{Par}}$. 
Remark 2.34. Let us consider two more types of points which can appear during the regeneration process:

(I) First, recall that each $y \in Y$ is a 2-point: it is on a line, which is the intersection of two planes. During the regeneration process, this line is regenerated into a conic. If $y$ is on the line $L_{i}$, whose corresponding edge in $T_{0}^{*}$ is $e_{i}$, then we induce the relation $\Gamma_{i}=\Gamma_{i^{\prime}}$ in $G$, where $\left\{\Gamma_{i}, \Gamma_{i^{\prime}}\right\}$ is the corresponding generators of $e_{i}$. Explicitly, the local fundamental group is $\left\{\Gamma_{i}, \Gamma_{i^{\prime}}: \Gamma_{i}=\right.$ $\left.\Gamma_{i^{\prime}}\right\} \simeq \mathbb{Z}$. This is due to the fact that the line $L_{i}$ is regenerated to a conic such that the branch point of the conic (which corresponds to $y$ ) induces the relation $\Gamma_{i}=\Gamma_{i^{\prime}}$.

(II) The second case is that $x \in\left\{p_{i}\right\}_{i=1}^{m^{\prime}}, x \notin Q$ and thus $x$ is a projection of a singular point of $R_{0}$ (if $x$ were not a projection of a singular point of $R_{0}$, then the projection would not be a generic one). Note that $v(x)>2$. Let us assume that the local configuration of lines exiting from $x$ is as in the following figure, when the lines are numerated by their order of appearance in the degeneration process:

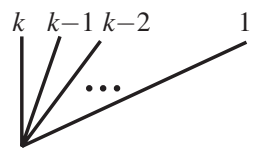

Figure 2.11. Local neighborhood of a $k$-point.

In this case, the local braid monodromy factorization was calculated in [12] and one can induce easily the local fundamental group associated for this point(see e.g. [23, Subsection 4.5]). Note that other numerations can appear also in non-planar degenerations, such as in the degeneration of $\mathbb{C P}^{1} \times C_{g}$ $(g \geq 1$. See Subsection 3.2 and Remark 3.10).

Remark 2.35. Recall that some of the singular points of a generic projection $B \rightarrow$ $\mathbb{C P}^{1}$ do not regenerate from $B_{0}$. By Condition (2) on $S$ (see Definition 2.15), these singular points would be branch points. These branch points only induce relations of the form $\left\{\Gamma_{j}=\Gamma_{j^{\prime}}\right\}$ when $\Gamma_{j}, \Gamma_{j^{\prime}}$ correspond to the same line $l_{j}$ in the degenerated branch curve $B_{0}$ (see [31] for further explanations).

We now examine what is the relation between the local fundamental groups $G_{x}$ and the group $G$. It is clear that for each $x, G_{x} \hookrightarrow G$, and in fact $G \simeq$ $\left(\underset{x \in P \cup Y}{*} G_{x}\right) /\left\langle R_{I}\right\rangle$ where $R_{I}$ is the identification of the same generators in $G$ belonging to different $G_{x}$ 's. Since we find a presentation of $G$ (and respectively of the groups $G_{x}$ ) by means of the Van-Kampen theorem, we can say that $G$ is generated by $2 l$ (respectively $2 v(x)$ ) generators. However, by the definition of $Q$ and $T_{0}^{*}$ the number of generators for $G$ can be reduced to $2(l-m)$.

Let us examine two cases:

(i) Assume that $\ell-m=n-1$. By definition, for each $x \in Q, G_{x}$ is isomorphic to a quotient of $\widetilde{B}_{v(x)}^{(2)}$ (where this $G_{x}$ is generated by $2(v(x)-1)$ generators $\left.\left\{\gamma_{x, i}, \gamma_{x, i^{\prime}}\right\}_{i=1}^{v(x)-1}\right)$. 
Lemma 2.36. Let $\Gamma \in G$ be a generator. So there exists $x \in Q$ s.t. $\Gamma$ is a generator of $G_{x}$.

Proof. Assume that there is a generator $\Gamma_{0}$ of $G$ such that it is not a generator of $G_{x}$ for every $x \in Q$. This generator corresponds to a line $l_{0}$ in $B_{0}$. By our construction, there are two points $p_{1}, p_{2}$ on $l_{0}$ that belong to the set $P^{\prime} \cup Y$, and by assumption, both of them do not belong to $Q$ (recall that $P^{\prime}$ is the set of singular points of $B_{0}$ which are images of singular points of $R_{0}$ and that $Y$ is the set of 2-points). We now look at two cases:

(I) One of the points belongs to $Y$.

Let $p_{1} \in Y, p_{2} \in P^{\prime}$. The point $p_{2}$ is an "inner" point (see Condition (4) in Definition 2.17), i.e., it does not lie on the border of the degenerated surface $S_{0}$, as in this case $l_{0}$ would not induce a generator (recall that we do not consider the border lines as part of $B_{0}$ ). Thus, the local neighborhood of $p_{1}, p_{2}$ in $S_{0}^{*}$ looks as in the following figure:

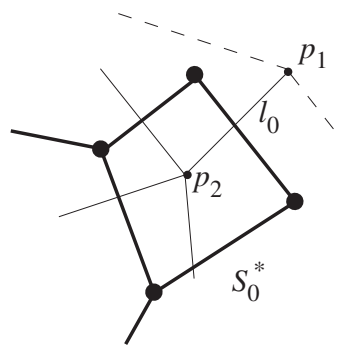

Figure 2.12. Local neighborhood of $p_{1}, p_{2}$.

Since there is a spanning subtree $T_{0}^{*}$ (by Lemma 2.30), one of the neighboring vertices to $p_{2}$ has to be in $Q$, as otherwise, in the process of the construction of $T_{0}^{*}$, we could not "terminate" the circle $C$ whose center is the point $p_{2}$. Denote this vertex by $p_{2}^{1}$ and delete an edge from the circle $C$ (see the figure below).

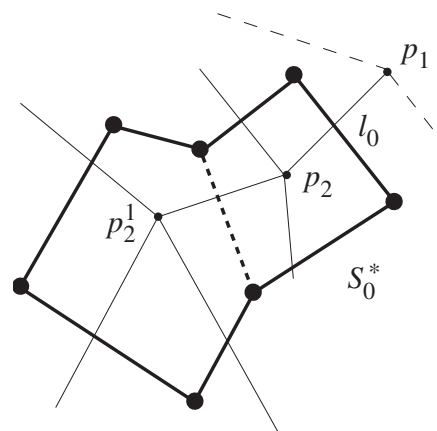

Figure 2.13. Local neighborhood of $p_{1}, p_{2}, p_{2}^{1}$. The dashed edge is the erased edge when trying to eliminate the circle containing $p_{2}$. 
However, now we have a new circle $C_{1}$ containing the points $p_{2}, p_{2}^{1}$. Thus there is another point $p_{2}^{2}$ in $Q$, neighbor to $p_{2}$ or to $p_{2}^{1}$, as we have to terminate the circle $C_{1}$, and we continue as above. But since this process is finite (there are finite number of points in $Q$ ), eventually we couldn't erase one of the edges from the circle $C_{j}$ (containing the points $p_{2}, p_{2}^{1}, \ldots, p_{2}^{j}$ ). This is due to the fact that we would not find "new" points in $Q$ s.t. one of the corresponding edges to them can be erased. Thus we get a contradiction.

(II) Two of the points belong to $P^{\prime}$. We get a contradiction as in the first case, since now we have two circles $C$ and $C^{\prime}$, each around every point, which eventually could not be resolved.

Thus the union of all the generators of these $G_{x}$ 's (s.t. we identify the same generators in $G)$ is the set of the $2(n-1)$ generators of $G$. We know that

$$
G \simeq\left(\left(\underset{x \in Q}{*} G_{x}\right) /\left\langle R_{I_{Q}}\right\rangle *\left(\underset{x \notin Q}{*} G_{x}\right)\right) /\left\langle R_{\mathrm{rest}}\right\rangle,
$$

Where $R_{I_{Q}}\left(R_{\text {rest }}\right)$ is the set of relations identifying identical generators in different local fundamental groups for $x \in Q$ (respectively the set of the other relations, e.g., induced from identifying identical generators in different local fundamental groups for $x \notin Q$, or from the local fundamental groups of parasitic intersection points or from extra branch points). But the generators of $G$ are the generators of $\underset{x \in Q}{*} G_{x}$, and thus

$$
G \simeq\left(\left(\underset{x \in Q}{*} G_{x}\right) /\left\langle R_{I_{Q}} \cup R_{\text {Par }}\right\rangle\right) /\left\langle R_{\text {rest' }}\right\rangle .
$$

Denoting $\left.G_{Q} \doteq \underset{x \in Q}{*} G_{x}\right) /\left\langle R_{I_{Q}} \cup R_{\text {Par }}\right\rangle$ it is enough to prove that there is an epimorphism $\widetilde{B}_{n}^{(2)} \rightarrow G_{Q}$.

Numerate the generators of $G_{Q}$ by $\left\{\Gamma_{i}, \Gamma_{i^{\prime}}\right\}_{i=1}^{n-1}$ associated to the edges $E_{T}=$ $\left\{t_{i}\right\}_{i=1}^{n-1}$ in the tree $T_{0}^{*}$, and let $\left\{x_{i}, x_{i^{\prime}}\right\}_{i=1}^{n-1}$ be the generators of $\widetilde{B}_{n}^{(2)}$. Define the epimorphic map

$$
\begin{gathered}
\alpha: \widetilde{B}_{n}^{(2)} \rightarrow G_{Q}, \\
x_{i} \mapsto \Gamma_{i}, x_{i^{\prime}} \mapsto \Gamma_{i^{\prime}}
\end{gathered}
$$

(possibly after conjugation. see Remark 2.32). We have to prove that the relations in $\widetilde{B}_{n}^{(2)}$ hold in $G_{Q}$. Since $G_{x} \simeq \widetilde{B}_{v(x)}^{(2)} / R_{v(x)}$ for each $x \in Q$ it is clear that the relations in $\widetilde{B}_{n}^{(2)}$ of the form $a b a=b a b$ hold in $G_{Q}$. The commutator relations which are not induced from the commutator relations in $\widetilde{B}_{v(x)}^{(2)}, x \in Q$ hold in $G_{Q}$ as the set of relations in $G_{Q}$ includes the set $R_{\mathrm{Par}}$.

(ii) Assume that $\ell-m<n-1$. Again, there exist $k \in \mathbb{N}, k<m$ such that $\ell-k=n-1$. Previously, in Lemma 2.30, we chose $k$ points from $Q$ to 
construct $T_{0}^{*}$. Therefore we can continue as above. Note that by Remark 2.31, even if the point $p_{2}$ (in Lemma 2.36) will have two neighboring vertices $\in Q$, we still could not resolve the circle $C$.

Remark 2.37. Recall that for a degeneratable surface $S$ that satisfies all the conditions, we denoted $n=\operatorname{deg} S, m=$ number of singular points $p$ of $B_{0}$ whose local fundamental group is a quotient of $\widetilde{B}_{v(p)}^{(2)}$, and by $\bar{m}$ the number of vertices in $\mathrm{Graph}_{S_{0}}$ (see Definition 2.11). By the restrictions imposed by Remark 2.14 and Theorem 2.22, we can bound $\ell=\frac{1}{2} \operatorname{deg} B$. Explicitly, for $B$ to be a branch of curve of degree $2 \ell$ of a embedded-degeneratable surface s.t. $G$ would be virtually solvable, the following inequalities should be satisfied:

$$
\max (n, \bar{m}+n)<\ell+1 \leq m+n .
$$

Remark 2.38. As can be seen from Subsection 2.3.1, Example 2.23 and Remark 2.37, the complete classification of smooth surfaces whose planar degeneration satisfies the condition introduced in Theorem 2.22 is not yet known, though some new restrictions are now clearer (e.g. inequality (2.1)). Moreover, [10, Section 8] has found some restrictions on surfaces admitting planar degeneration with some specific conditions on the singularities of the degenerated surface. These conditions do shed some light on our class of surfaces. For example, every singular point in the degenerated surface, denoted in [10, Definition 3.5] as $E_{m}$-point $(m>3)$, belongs to the set $Q$ (see Definition 2.9). Given a planar degeneration, [10, Theorem 8.4] imposes conditions on the square of the canonical class of the surface, when the degenerated surface has some specific singular points. Certainly this theorem can be generalized to include more cases of singular points in the set $Q$ and to the bigger classes of embedded-degeneratable surfaces. Moreover, [10, Proposition 8.6] states that for every surface there might be a birational model of it that is degeneratable into a union of planes with mild singularities $p_{i}$ (s.t. the local fundamental group $G_{p_{i}}$ is known), though it is not clear whether if this model is even simplydegeneratable (see Dentition 2.15).

Note also that all the surfaces in Example 2.23 are simply connected, and this raises the conjecture whether the desired class of surfaces is contained in the class of simply connected surfaces. Indeed, this is supported by that fact that if $S$ is a surface s.t. $\pi_{1}(S)$ contains a free group of rank 2, then $S$ does not satisfy the condition in Theorem 2.22 (as $G$ is not virtually solvable). However, this is the subject of an ongoing research.

\section{Non simply connected scrolls}

By [20, Proposition 4.13], for a projective complex surface $S$, if $\pi_{1}(S)$ is not virtually solvable, then $\pi_{1}\left(\mathbb{C P}^{2}-B\right)$ is not virtually solvable, where $B$ is the branch curve of $S$ with respect to a generic projection. As Liedtke [20] points out, for $S$ a ruled surface over a curve of genus bigger than $1, \pi_{1}(S)$ contains a free group 
of rank 2. Therefore, for such an $S$, there does not exist a planar degeneration with enough "good" singular points (i.e. points in the set $Q$. See definition 2.9). However, in the next section we examine what would be a possible structure for $G=\pi_{1}\left(\mathbb{C}^{2}-B\right)$ for such a surface. Specifically, we consider the structure of this group when the set $Q$ is empty.

By Conjecture 2.25 , the existence of points in the set $Q$ would imply that $G$ would be a quotient of $\widetilde{A}(T)^{(2)}$, where as in our case (see Thereom 3.35), $G$ is a quotient of $A(T)$ (where $T$ is an associated graph to the degeneration of $S$ ), as in Example 2.28(1). This strengthens Conjecture 2.26.

For the convenience of the reader, we begin with recalling the notions of the Braid Monodromy Factorization (BMF) in subsection 3.1. We then investigate the surface $\mathbb{C P}^{1} \times C_{g}$, where $C_{g}$ is a curve of genus $g \geq 1$, and the corresponding fundamental group $\pi_{1}\left(\mathbb{C}^{2}-B_{g}\right)$, in subsections 3.2 and 3.3. Using the results, we compute the fundamental group of the Galois cover of these surfaces in Subsection 3.4.

\subsection{Background on braid monodromy factorization}

Recall that computing the braid monodromy is the main tool to compute fundamental groups of complements of curves. The reader who is familiar with this subject can skip the following definitions to Subsection 3.2. We begin by defining the braid monodromy associated to a curve.

Let $D$ be a closed disk in $\mathbb{R}^{2}, K \subset \operatorname{Int}(D), K$ finite, $n=\# K$. Recall that the braid group $B_{n}(D, K)$ can be defined as the group of all equivalent diffeomorphisms $\beta$ of $D$ such that $\beta(K)=K,\left.\beta\right|_{\partial D}=\left.\mathrm{Id}\right|_{\partial D}$ (two diffeomorphisms are equivalent if they induce the same automorphism on $\pi_{1}(D-K, u)$ ).

Definition 3.1. $H(\sigma)$ is a half-twist defined by $\sigma$.

Let $a, b \in K$, and let $\sigma$ be a smooth simple path in $\operatorname{Int}(D)$ connecting $a$ with $b$ s.t. $\sigma \cap K=\{a, b\}$. Choose a small regular neighborhood $U$ of $\sigma$ contained in $\operatorname{Int}(D)$, s.t. $U \cap K=\{a, b\}$. Denote by $H(\sigma)$ the diffeomorphism of $D$ which switches $a$ and $b$ by a counterclockwise $180^{\circ}$ rotation and is the identity on $D \backslash U$. Thus it defines an element of $B_{n}[D, K]$, called the half-twist defined by $\sigma$.

Denote $[A, B]=A B A^{-1} B^{-1},\langle A, B\rangle=A B A B^{-1} A^{-1} B^{-1}$. We recall Artin's presentation of the braid group:

Theorem 3.2. $B_{n}$ is generated by the half-twists $H_{i}$ of a sequence of paths $\sigma_{i=1}^{n-1}$ (such that $\sigma_{i}$ connected the $i^{\text {th }}$ and the $(i+1)^{\text {th }}$ points) and all the relations between $H_{1}, \ldots, H_{n-1}$ follow from:

$$
\begin{aligned}
& {\left[H_{i}, H_{j}\right]=1 \text { if }|i-j|>1} \\
& \left\langle H_{i}, H_{j}\right\rangle=1 \text { if }|i-j|=1 .
\end{aligned}
$$

Assume that all of the points of $K$ are on the $X$-axis (when considering $D$ in $\mathbb{R}^{2}$ ). In this situation, if $a, b \in K$, and $z_{a, b}$ is a path that connects them, then we denote the corresponding half-twist by $Z_{a, b}=H\left(z_{a, b}\right)$. If $z_{a, b}$ is a path that goes below the 
$X$-axis, then we denote it by $\underline{Z}_{a, b}$, or just $Z_{a, b}$. If $z_{a, b}$ is a path that goes above the $x$-axis, then we denote it by $\bar{Z}_{a, b}$. We also denote by $\underline{\underline{Z}}_{a, b} \underset{(c-d)}{\left(\bar{Z}_{a, b}\right)}$ the braid induced from a path connecting the points $a$ and $b$ below (respectively above) the $X$-axis, going above (respectively below) it from the point $c$ till point $d$.

Definition 3.3. The braid monodromy with respect to $C, \pi, u$. Let $C$ be a curve, $C \subseteq \mathbb{C}^{2}$. Choose $O \in \mathbb{C}^{2}, O \notin C$ such that the projection $f: \mathbb{C}^{2} \rightarrow \mathbb{C}^{1}$ with center $O$ will be generic when restricting it to $C$. We denote $\pi=\left.f\right|_{C}$ and $\operatorname{deg} \pi=\operatorname{deg} C$ by $m$. Let $N=\left\{x \in \mathbb{C}^{1} \mid \# \pi^{-1}(x)<m\right\}$. Take $u \notin N$, and let $\mathbb{C}_{u}^{1}=f^{-1}(u)$. There is a naturally defined homomorphism describing the motion of the points in the fiber

$$
\pi_{1}\left(\mathbb{C}^{1}-N, u\right) \stackrel{\varphi}{\rightarrow} B_{m}\left[\mathbb{C}_{u}^{1}, \mathbb{C}_{u}^{1} \cap C\right]
$$

which is called the braid monodromy with respect to $C, \pi, u$, where $B_{m}$ is the braid group.

In fact, denoting by $E$ a big disk in $\mathbb{C}^{1}$ s.t. $E \supset N$, we can also take the path in $E \backslash N$ not to be a loop, but just a non-self-intersecting path. This induces a diffeomorphism between the models $(D, K)$ at the two ends of the considered path, where $D$ is a big disk in $\mathbb{C}_{u}^{1}$, and $K=\mathbb{C}_{u}^{1} \cap C \subset D$.

Definition 3.4. $\psi_{T}$ the Lefschetz diffeomorphism induced by a path $T$. Let $T$ be a path in $E \backslash N$ connecting $x_{0}$ with $x_{1}, T:[0,1] \rightarrow E \backslash N$. There exists a continuous family of diffeomorphisms $\psi_{(t)}: D \rightarrow D, t \in[0,1]$, such that $\psi_{(0)}=I d$, $\psi_{(t)}\left(K\left(x_{0}\right)\right)=K(T(t))$ for all $t \in[0,1]$, and $\psi_{(t)}(y)=y$ for all $y \in \partial D$. For emphasis we write $\psi_{(t)}:\left(D, K\left(x_{0}\right)\right) \rightarrow(D, K(T(t))$. A Lefschetz diffeomorphism induced by a path $T$ is the diffeomorphism

$$
\psi_{T}=\psi_{(1)}:\left(D, K\left(x_{0}\right)\right) \underset{\sim}{\sim}\left(D, K\left(x_{1}\right)\right) .
$$

Since $\psi_{(t)}\left(K\left(x_{0}\right)\right)=K(T(t))$ for all $t \in[0,1]$, we have a family of canonical isomorphisms

$$
\psi_{(t)}^{\nu}: B_{p}\left[D, K\left(x_{0}\right)\right] \underset{\sim}{\longrightarrow} B_{p}[D, K(T(t))], \quad \text { for all } t \in[0,1] .
$$

We recall Artin's theorem on the presentation of the Dehn twist of the braid group as a product of braid monodromy elements of a geometric-base (a base of $\pi_{1}=$ $\pi_{1}\left(\mathbb{C}^{1}-N, u\right)$ with certain properties; see [26] for definitions).

Theorem 3.5. Let $C$ be a curve transversal to the line in infinity, and $\varphi$ is a braid monodromy of $C, \varphi: \pi_{1} \rightarrow B_{m}$. Let $\delta_{i}$ be a geometric (free) base (called a g-base) of $\pi_{1}$, and $\Delta^{2}$ is the generator of Center $\left(B_{m}\right)$. Then:

$$
\Delta^{2}=\prod \varphi\left(\delta_{i}\right) .
$$

This product is also defined as the braid monodromy factorization (BMF) related to a curve $C$. 
Note that if $x_{1}, \ldots, x_{n-1}$ are the generators of $B_{n}$, then we know that $\Delta^{2}=$ $\left(x_{1} \cdot \ldots \cdot x_{n-1}\right)^{n}$ and thus $\operatorname{deg}\left(\Delta^{2}\right)=n(n-1)$.

So in order to find out what is the braid monodromy factorization of $\Delta_{p}^{2}$, we have to find out what are $\varphi\left(\delta_{i}\right), \forall i$. We refer the reader to the definition of a skeleton (see [27]) $\lambda_{x_{j}}, x_{j} \in N$, which is a model of a set of paths connecting points in the fiber, s.t. all those points coincide when approaching $A_{j}=\left(x_{j}, y_{j}\right) \in C$, when we approach this point from the right. To describe this situation in greater detail, for $x_{j} \in N$, let $x_{j}^{\prime}=x_{j}+\alpha$. So the skeleton in $x_{j}$ is defined as a system of paths connecting the points in $K\left(x_{j}^{\prime}\right) \cap D\left(A_{j}, \varepsilon\right)$ when $0<\alpha \ll \varepsilon \ll 1, D\left(A_{j}, \varepsilon\right)$ is a disk centered in $A_{j}$ with radius $\varepsilon$.

For a given skeleton, we denote by $\Delta\left\langle\lambda_{x_{j}}\right\rangle$ the braid by rotates by 180 degrees counterclockwise a small neighborhood of the given skeleton. Note that if $\lambda_{x_{j}}$ is a single path, then $\Delta\left\langle\lambda_{x_{j}}\right\rangle=H\left(\lambda_{x_{j}}\right)$.

We also refer the reader to the definition of $\delta_{x_{0}}$, for $x_{0} \in N$ (see [27]), which describes the Lefschetz diffeomorphism induced by a path going below $x_{0}$, for different types of singular points (tangent, node, branch; for example, when going below a node, a half-twist of the skeleton occurs and when going below a tangent point, a full-twist occurs).

We define, for $x_{0} \in N$, the following number: $\varepsilon_{x_{0}}=1,2,4$ when $\left(x_{0}, y_{0}\right)$ is a branch/node/tangent point (respectively). Explicitly, in local coordinates $(x, y)$ (where $\left(x_{0}, y_{0}\right)=(0,0)$ ), a branch is a singular point (with respect to the projection) with local equation $y^{2}=x$, a node $-y^{2}=x^{2}$, and a tangent $y\left(y-x^{2}\right)=0$. So we have the following statement (see [27, Proposition 1.5]):

Let $\gamma_{j}$ be a path below the real line from $x_{j}$ to $u$, s.t. $\ell\left(\gamma_{j}\right)=\delta_{j}$. So

$$
\varphi_{u}\left(\delta_{j}\right)=\varphi\left(\delta_{j}\right)=\Delta\left\langle\left(\lambda_{x_{j}}\right)\left(\prod_{m=j-1}^{1} \delta_{x_{m}}\right)\right\rangle^{\varepsilon_{x_{j}}} .
$$

When denoting $\xi_{x_{j}}=\left(\lambda_{x_{j}}\right)\left(\prod_{m=j-1}^{1} \delta_{x_{m}}\right)$ we get

$$
\varphi\left(\delta_{j}\right)=\Delta\left\langle\left(\xi_{x_{j}}\right)\right\rangle^{\varepsilon_{x_{j}}} .
$$

Note that the last formula gives an algorithm to compute the needed factorization. For a detailed explanation of the braid monodromy, see [26].

Assume that we have a curve $\bar{C}$ in $\mathbb{C P}^{2}$ and its BMF. Then we can calculate the groups $\pi_{1}\left(\mathbb{C P}^{2}-\bar{C}\right)$ and $\pi_{1}\left(\mathbb{C}^{2}-C\right)$ (where $\left.C=\bar{C} \cap \mathbb{C}^{2}\right)$. Recall that a $g$-base is an ordered free base of $\pi_{1}(D \backslash F, v)$, where $D$ is a closed disc, $F$ is a finite set in $\operatorname{Int}(D), v \in \partial D$ which satisfies several conditions; see $[26,27]$ for the explicit definition.

Let $\left\{\Gamma_{i}\right\}$ be a $g$-base of $G=\pi_{1}\left(\mathbb{C}_{u}-\left(\mathbb{C}_{u} \cap C\right), u\right)$, where $\mathbb{C}_{u}=\mathbb{C} \times u$. We cite now the Zariski-Van Kampen Theorem (for cuspidal curves) in order to compute the relations between the generators in $G$. 
Theorem 3.6 (Zariski-Van Kampen (cuspidal curves version)). Let $\bar{C}$ be a cuspidal curve in $\mathbb{C P}^{2}$. Let $C=\mathbb{C}^{2} \cap \bar{C}$. Let $\varphi$ be a braid monodromy factorization with respect to $C$ and $u$. Let $\varphi=\prod_{j=1}^{p} V_{j}^{v_{j}}$, where $V_{j}$ is a half-twist and $v_{j}=1,2,3$.

For every $j=1 \ldots p$, let $A_{j}, B_{j} \in \pi_{1}\left(\mathbb{C}_{u}-C, u\right)$ be such that $A_{j}, B_{j}$ can be extended to a g-base of $\pi_{1}\left(\mathbb{C}_{u}-C, u\right)$ and $\left(A_{j}\right) V_{j}=B_{j}$. Let $\left\{\Gamma_{i}\right\}$ be a g-base of $\pi_{1}\left(\mathbb{C}_{u}-C, u\right)$ corresponding to the $\left\{A_{i}, B_{i}\right\}$, where $A_{i}, B_{i}$ are expressed in terms of $\Gamma_{i}$. Then $\pi_{1}\left(\mathbb{C}^{2}-C, u\right)$ is generated by the images of $\left\{\Gamma_{i}\right\}$ in $\pi_{1}\left(\mathbb{C}^{2}-C, u\right)$ and the only relations are those implied from $\left\{V_{j}^{v_{j}}\right\}$, as follows:

$$
\begin{cases}A_{j} \cdot B_{j}^{-1} & \text { if } \quad v_{j}=1 \\ {\left[A_{j}, B_{j}\right]=1} & \text { if } \quad v_{j}=2 \\ \left\langle A_{j}, B_{j}\right\rangle=1 & \text { if } \quad v_{j}=3 .\end{cases}
$$

$\pi_{1}\left(\mathbb{C P}^{2}-\bar{C}, *\right)$ is generated by $\left\{\Gamma_{i}\right\}$ with the above relations and one more relation $\prod_{i} \Gamma_{i}=1$.

The following figure illustrates how to find $A_{i}, B_{i}$ from the half-twist $V_{i}=H(\sigma)$ :
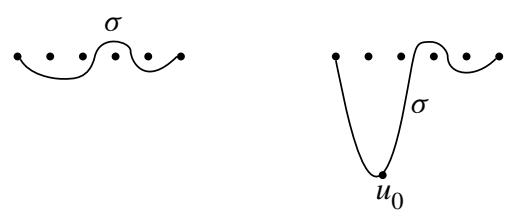
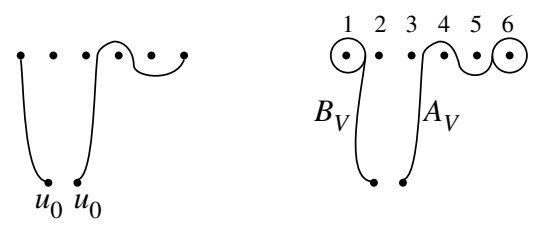

Figure 3.1.

So

$$
A_{V}=\Gamma_{4}^{-1} \Gamma_{6} \Gamma_{4}, B_{V}=\Gamma_{1} .
$$

See [30] on how to induce the expressions corresponding to $A_{V}, B_{V}$.

\subsubsection{Example of a $B M F$}

We give here an example of computing a simple Braid Monodromy Factorization, for the following configuration:

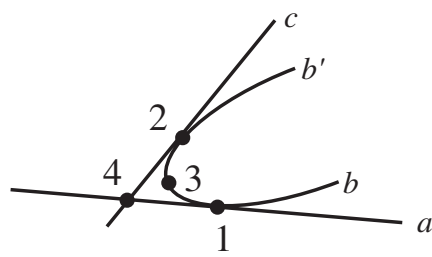

Figure 3.2. 
We will need this factorization in Subsection 3.2, where it will be the factorization of the first regeneration a certain singular point.

Proposition 3.7. The local braid monodromy factorization of the above configuration is

$$
\varphi=Z_{a b}^{4} Z_{b^{\prime} c}^{4} \widetilde{Z}_{b b^{\prime}} \widetilde{Z}_{a c}^{2}
$$

where the braids $\widetilde{Z}_{b b^{\prime}}, \widetilde{Z}_{a c}$ correspond to the following paths:
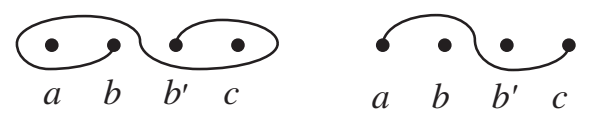

Figure 3.3.

Proof. Let $\left\{p_{j}\right\}_{j=1}^{4}$ be the singular points of the above configuration with respect to $\pi_{1}$ (the projection to the $X$-axis) as follows:

$p_{1}, p_{2}$ - the tangent points of the parabola and the lines $L_{a}, L_{c}$ (denoted by $a$ and $c$ in Figure 3.2).

$p_{3}$ - the branch point of the parabola.

$p_{4}$ - the intersection point of $L_{a}, L_{c}$.

Let $E$ (respectively $D$ ) be a closed disk on the $X$-axis (respectively $Y$-axis). Let $N=\left\{x\left(p_{j}\right)=x_{j} \mid 1 \leq j \leq 4\right\}$, s.t. $N \subset E-\partial E$. Let $M$ be a real point on the $x$-axis, s.t. $x_{j} \ll M, \forall x_{j} \in N, 1 \leq j \leq 4$. There is a $g$-base $\ell\left(\gamma_{j}\right)_{j=1}^{4}$ of $\pi_{1}(E-N, u)$, s.t. each path $\gamma_{j}$ is below the real line and the values of $\varphi_{M}$ with respect to this base and $E \times D$ are the ones given in the proposition. We look for $\varphi_{M}\left(\ell\left(\gamma_{j}\right)\right)$ for $j=1, \cdots, 4$. Choose a $g$-base $\ell\left(\gamma_{j}\right)_{j=1}^{4}$ as above and put all the data in the following table:

\begin{tabular}{c|c|c|c}
$j$ & $\lambda_{j}$ & $\varepsilon_{j}$ & $\delta_{j}$ \\
\hline 1 & $\langle a, b\rangle$ & 4 & $\Delta^{2}\langle a, b\rangle$ \\
2 & $\left\langle b^{\prime}, c\right\rangle$ & 4 & $\Delta^{2}\left\langle b^{\prime}, c\right\rangle$ \\
3 & $\left\langle b, b^{\prime}\right\rangle$ & 1 & $\Delta_{I R}^{1 / 2}\langle b\rangle$ \\
4 & $\langle a, c\rangle$ & 2 & -
\end{tabular}

So, we get the following:

$$
\begin{aligned}
& \xi_{x_{1}}=z_{a, b}, \varphi_{M}\left(\ell\left(\gamma_{1}\right)\right)=Z_{a b}^{4} \\
& \xi_{x_{2}}=z_{b^{\prime}, c}, \varphi_{M}\left(\ell\left(\gamma_{2}\right)\right)=Z_{b^{\prime} c}^{4}
\end{aligned}
$$

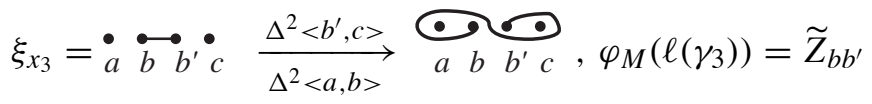

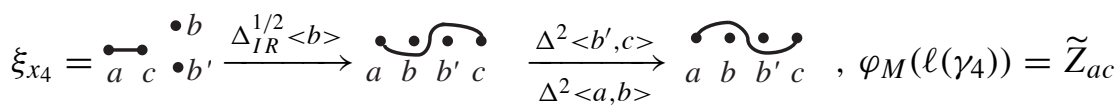




\subsubsection{Regeneration rules}

We finish this subsection with the regeneration rules. Given a degeneration $\rho$ : $S \rightarrow \Delta$, the regeneration rules explain how the braid monodromy factorization of the branch curve of $S_{0}$ (under generic projection) changes when passing to the braid monodromy factorization of the branch curve of $S_{t}, t \neq 0$. The rules are (see [29, pages 336-337]):

1. First regeneration rule: The regeneration of a branch point of any conic:

A factor of the braid monodromy of the form $Z_{i, j}$ is replaced in the regeneration by $Z_{i^{\prime}, j} \cdot \stackrel{(j)}{Z}_{i, j^{\prime}}$.

2. Second regeneration rule: The regeneration of a node:

A factor of the form $Z_{i j}^{2}$ is replaced by a factorized expression $Z_{i i^{\prime}, j}^{2}:=Z_{i^{\prime} j}^{2} \cdot Z_{i j}^{2}$, $Z_{i, j j^{\prime}}^{2}:=Z_{i j^{\prime}}^{2} \cdot Z_{i j}^{2}$ or by $Z_{i i^{\prime}, j j^{\prime}}^{2}:=Z_{i^{\prime} j^{\prime}}^{2} \cdot Z_{i j^{\prime}}^{2} Z_{i^{\prime} j}^{2} \cdot Z_{i j}^{2}$.

3. Third regeneration rule: The regeneration of a tangent point:

A factor of the form $Z_{i j}^{4}$ in the braid monodromy factorized expression is replaced by $Z_{i, j j^{\prime}}^{3}:=\left(Z_{i j}^{3}\right)^{Z} j^{\prime} \cdot\left(Z_{i j}^{3}\right) \cdot\left(Z_{i j}^{3}\right)^{Z_{j j^{\prime}}^{-1}}$

\subsection{The fundamental group related to $\mathbb{C} \mathbb{P}^{1} \times C_{1}$}

We start by analyzing the degeneration of the surface $\mathbb{C P}^{1} \times C_{1}$, where $C_{1}$ is a smooth curve of genus 1. Although this surface was already investigated in [7], we present here a different degeneration, which can be generalized to surfaces of the form $\mathbb{C P}^{1} \times C_{g}\left(C_{g}\right.$ is a smooth genus- $g$ curve $)$. This generalization will be discussed in the next subsection but we give here a rough description of how this degeneration is done. See also Construction 3.27.

Construction 3.8. We review the degeneration described in [9]. Let $C$ be a smooth, rational normal curve of degree $n$ in $\mathbb{C P}^{n}$. Since $C$ degenerates to a union of $n$ lines $l_{i}$ (s.t. $l_{i} \cap l_{i+1}=p t$. for $1 \leq i \leq n-1, l_{i} \cap l_{j}=\emptyset$ for $|i-j|>1$ ), the smooth rational normal scroll $S=C \times \mathbb{C P}^{1} \subset \mathbb{C P}^{2 n+1}$ degenerates to surface $S^{\prime}=\bigcup_{i=1}^{n} S_{i}$ such that each $S_{i}$ is a quadric (i.e. isomorphic to $\mathbb{C P}^{1} \times \mathbb{C P}^{1}$ ). Each quadric $S_{i}$ meets $S-S_{i}$ either along one or two lines of the same ruling. Thus each quadric $S_{i}$ degenerates to the union of two planes meeting along a line $l_{i}$, leaving the other line(s) fixed. Therefore, in $\mathbb{C P}^{2 n+1}$, the scroll $S$ degenerates to a planar surface $S^{\prime \prime}$ of degree $2 n$. Assume $n>2$ Choose now two disjoint lines $\ell_{1}, \ell_{4}$ in the planes $S_{1}$ and $S_{4}$ such that $S_{1} \cap S_{2} \cap S_{3} \notin \ell_{1}, S_{3} \cap S_{4} \cap S_{5} \notin \ell_{4}$. As $\ell_{1}, \ell_{4}$ are skew, they span a $\mathbb{C P}^{3}$ which we denote as $\Pi$, such that $\Pi \cap S^{\prime \prime}=\ell_{1} \cup \ell_{4}$. Thus there exists a smooth quadric $Q$ in $\Pi$ such that $\ell_{1}, \ell_{4}$ are lines of the same ruling on $Q$ and $Q \cap S^{\prime \prime}=\ell_{1} \cup \ell_{4}$. There, in $\Pi, Q$ degenerates to two planes $P_{1}, P_{4}$ s.t. $\ell_{i} \in P_{i}$. In [9, Construction 4.2] one proves that the planar surface $S^{\prime \prime} \cap P_{1} \cap P_{4}$ is indeed a degeneration $\mathbb{C P}^{1} \times C_{1}$. See Figure 3.4 for the final degeneration when 
$\mathbb{C P}^{1} \times \mathbb{C P}^{1}$ is embedded with respect to the linear system $(1,3)$ (i.e. $n=3$ in the above notation).

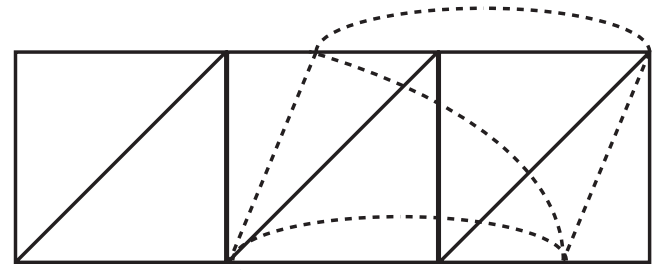

Figure 3.4. The degeneration of $\mathbb{C P}^{1} \times C_{1}$.

The dashed lines represent the attached degenerated quadric. Some of the planes are intersecting other planes in lines. We numerate (according to [26]) the singular points of this arrangement of lines by $V_{i}, 1 \leq i \leq 8$ and the lines of intersection by $L_{i}, 1 \leq i \leq 8$, as follows:

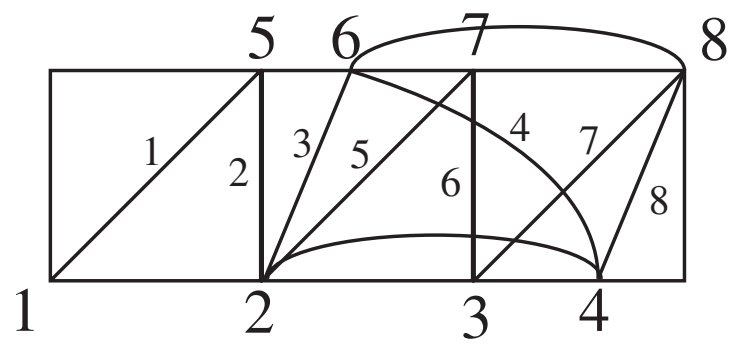

Figure 3.5. The numeration of the singular points (the larger numbers) and lines (the smaller numbers) of the degenerated ramification curve of $\mathbb{C P}^{1} \times C_{1}$.

Note that $\cup L_{i}$ is the degenerated ramification curve $R_{0}$ with respect to a generic projection. Thus, projecting the degenerated surface to $\mathbb{C P}^{2}$, we denote by $B_{0}$ the (degenerated) branch curve and the images of $V_{i}$ by $v_{i}$. We numerate the lines composing $B_{0}$ as before. Note that we have new singular points, beside the points $v_{i}$, called parasitic intersection (see subsection 2.4.1). These points are created from lines that do not intersect in $\mathbb{C P}^{9}$ but do intersect in $\mathbb{C P}^{2}$. The braid monodormy factorization of the degenerated branch curve is known to be (see [26]) $\Pi_{i=8}^{1} \widetilde{C}_{i} \Delta_{i}^{2}$, where $\widetilde{C}_{i}$ denotes the local braid monodromy factorization around the parasitic intersection points and $\Delta_{i}^{2}$ the local braid monodromy factorization around the point $v_{i}$. One can find the $\widetilde{C}_{i}$ 's according to [26, Theorem IX].

Remark 3.9. Since the regeneration of $\Delta_{i}^{2}$ for the different points was already done, we give here references to the final results. The points $v_{i}, i=3, . ., 8$ are 3 -points. (i.e., they are the images of the points $v_{i}$ which are locally the intersection of three planes. see e.g., [29]) The factors that they contribute to the factorization (i.e. their local BMFs) are either $Z_{a^{\prime}, b b^{\prime}}^{(3)} \cdot \widetilde{Z}_{a a^{\prime}}$ or $Z_{a a^{\prime}, b}^{(3)} \cdot \widetilde{Z}_{b b^{\prime}}\left(\right.$ where $\left.v_{i}=L_{a} \cap L_{b}\right)$. The point $v_{1}$ is a 2 -point and contributes to the factorization the factor $Z_{1,1^{\prime}}$ (see 
Notation 2.7 and Remark 2.34(I)). For the point $v_{2}$, see a more explicit explanation in the next remark.

Remark 3.10. In a small neighborhood of $v_{2}$, the first line that regenerates is $L_{3}$, which turns into a conic (see [29]). The braid monodromy factorization of this first regeneration is presented in Proposition 3.7. In the following regenerations we use the regeneration rules (see Subsection 3.1.2): the tangent points (i.e., a braid of the form $Z_{\ldots}^{4}$ ) are regenerated into three cusps (three braids of the form $Z_{\ldots}^{3}$ ) and a node (a braid of the form $Z_{\ldots . .}^{2}$ ) into four nodes. Explicitly, the factorization $Z_{23}^{4} Z_{3^{\prime} 5}^{4} \widetilde{Z}_{33^{\prime}} \widetilde{Z}_{25}^{2}$ is replaced by the factorization $Z_{22^{\prime}, 3}^{(3)} Z_{3^{\prime}, 55^{\prime}}^{(3)} \widetilde{Z}_{33^{\prime}} \underline{(3)}_{22^{\prime}, 55^{\prime}}$.

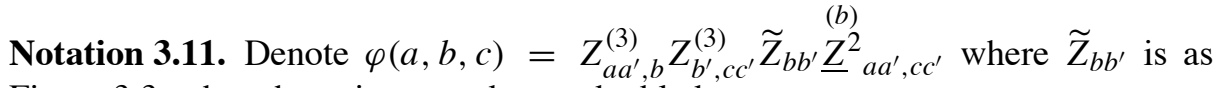
Figure 3.3, when the points $a$ and $c$ are doubled.

Notation 3.12. $B_{1}=$ the branch curve of $\mathbb{C P}^{1} \times C_{1}$ embedded in $\mathbb{C P}^{9}$ with respect to a generic projection.

From Remarks 3.10 and 3.9, we can induce the BMF of $B_{1}$ :

Theorem 3.13. The braid monodromy factorization of the branch curve $B_{1}$ of a generic projection of $\mathbb{C P} \mathbb{P}^{1} \times C_{1}$ embedded in $\mathbb{C P}$ is:

$$
\Delta^{2}=\prod_{i=8}^{1} C_{i} \cdot H_{i},
$$

where

$$
C_{i}=\mathrm{id}, i=1,5, . ., 8, \quad C_{2}=D_{3} \cdot D_{5}, \quad C_{3}=D_{6} \cdot D_{7}, \quad C_{4}=D_{4} \cdot D_{8}
$$

where

$$
\begin{array}{lll}
D_{3}=\bar{Z}_{11^{\prime}, 33^{\prime}}^{2}, & D_{4}=\underline{Z}_{11^{\prime}, 44^{\prime}}^{2} \cdot Z_{22^{\prime}, 44^{\prime}}^{2}, & D_{5}=\bar{Z}_{11^{\prime}, 55^{\prime}}^{2} \cdot Z_{44^{\prime}, 55^{\prime}}^{2}, \\
D_{6}=\prod_{i=1}^{4} \bar{Z}_{i i^{\prime}, 66^{\prime}}^{2}, & D_{7}=\Pi_{i=1}^{5} \bar{Z}_{i i^{\prime}, 77^{\prime}}^{2}, & D_{8}=\prod_{\substack{i=1,2,2 \\
3,5,6}} \bar{Z}_{i i^{\prime}, 88^{\prime}}^{2}
\end{array}
$$

and

$H_{1}=Z_{1,1^{\prime}}, H_{i}=Z_{a^{\prime}, b b^{\prime}}^{(3)} \cdot \widetilde{Z}_{a a^{\prime}}$ for $i=4,5,7,8, H_{i}=Z_{a a^{\prime}, b}^{(3)} \cdot \widetilde{Z}_{b b^{\prime}}$ for $i=3,6$

(when $v_{i}=L_{a} \cap L_{b}, a<b$ ), where $\widetilde{Z}_{., .}$is the braid induced from the following motion: $\widetilde{Z}_{a a^{\prime}}:$

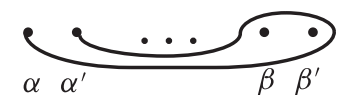


$\widetilde{Z}_{b b^{\prime}}:$

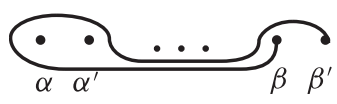

$$
Z_{a^{\prime}, b b^{\prime}}^{(3)}=\prod_{q=-1,0,1}\left(Z_{a^{\prime}, b}^{3}\right)_{b, b^{\prime}}^{q}, \quad Z_{a a^{\prime}, b}^{(3)}=\prod_{q=-1,0,1}\left(Z_{a^{\prime}, b}^{3}\right)_{Z_{a, a^{\prime}}^{q}}
$$

and $H_{2}=\varphi(2,3,5)$ where $\widetilde{Z}_{33^{\prime}}$ (a factor in the factorization $H_{2}$ ) is the braid induced from the following motion:

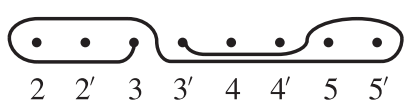

We recall the definition of an equivalence relation on the braid monodromy factorization. Let $H$ be a group.

Definition 3.14 (Hurwitz moves). Let $\vec{t}=\left(t_{1}, \ldots, t_{m}\right) \in H^{m}$. We say that $\vec{s}=$ $\left(s_{1}, \ldots, s_{m}\right) \in H^{m}$ is obtained from $\vec{t}$ by the Hurwitz move $R_{k}$ (or $\vec{t}$ is obtained from $\vec{s}$ by the Hurwitz move $R_{k}^{-1}$ ) if

$$
s_{i}=t_{i} \quad \text { for } i \neq k, k+1, s_{k}=t_{k} t_{k+1} t_{k}^{-1}, s_{k+1}=t_{k} .
$$

Definition 3.15 (Hurwitz move on a factorization). Let $H$ be a group $t \in H$. Let $t=t_{1} \cdot \ldots \cdot t_{m}=s_{1} \cdot \ldots \cdot s_{m}$ be two factorized expressions of $t$. We say that $s_{1} \cdot \ldots \cdot s_{m}$ is obtained from $t_{1} \cdot \ldots \cdot t_{m}$ by a Hurwitz move $R_{k}$ if $\left(s_{1}, \ldots, s_{m}\right)$ is obtained from $\left(t_{1}, \ldots, t_{m}\right)$ by a Hurwitz move $R_{k}$.

Definition 3.16. (1) Two factorizations are Hurwitz equivalent if they are obtained from each other by a finite sequence of Hurwitz moves.

(2) Let $g=g_{1} \cdot \ldots \cdot g_{n}$ be a factorized expression in a group $H\left(g_{i} \in H\right)$, and denote by ()$_{h}$ the conjugation by $h \in H$. We say that $g$ is invariant under $h$ if $g_{h} \doteq\left(g_{1}\right)_{h} \cdot \ldots \cdot\left(g_{n}\right)_{h}$ is Hurwitz equivalent to $g$.

Let us examine the invariance relations on the braid monodromy factorization from Theorem 3.13. From [29] we know that the expressions $C_{i}, 1 \leq i \leq 8$ and $H_{j}, 1 \leq j \leq 8, j \neq 2$ are invariant under $Z_{k k^{\prime}}^{q}, q \in \mathbb{Z}, k=1,4,6,7,8$. Recall also that the expressions of the form $Z_{i i^{\prime}, j j^{\prime}}^{2}$ are invariant under $Z_{i i^{\prime}}^{p} Z_{j j^{\prime}}^{q}$ and $Z_{i, j j^{\prime}}^{(3)}$ is invariant under $Z_{j j^{\prime}}^{k}\left(k, p, q \in \mathbb{Z}\right.$ ). Note that if $\sigma \cap\left[j, j^{\prime}\right]=\emptyset$ (where $\sigma$ is a path in a disc containing the points $j, j^{\prime}$ and $\left[j, j^{\prime}\right]$ is a line connecting $j$ and $j^{\prime}$ ) then $H(\sigma)$ is invariant under $Z_{j j^{\prime}}^{k}(k \in \mathbb{Z})$.

Remark 3.17. Using these rules, we see that $H_{2}$ is invariant under $Z_{22^{\prime}}^{p} Z_{55^{\prime}}^{q}$, and therefore the whole factorization is invariant under $Z_{11^{\prime}}^{p_{1}} Z_{22^{\prime}}^{p_{2}} \Pi_{j=4}^{8} Z_{j j^{\prime}}^{p_{j}}, p_{j} \in \mathbb{Z}$. 
As was explained, during the regeneration process, every generator $\Gamma_{j}$ is doubled into two generators: $\Gamma_{j}$ and $\Gamma_{j^{\prime}}$, so $\pi_{1}\left(\mathbb{C}^{2}-B_{1}\right)$ is generated by $\left\{\Gamma_{j}, \Gamma_{j^{\prime}}\right\}_{j=1}^{8}$. From now on, we denote the generator $\Gamma_{j}$ by $j$ and the generator $\Gamma_{j^{\prime}}$ by $j^{\prime}$. Let $\underline{j}$ denote $j$ or $j^{\prime}$, and $e$ the unit element in $\pi_{1}\left(\mathbb{C}^{2}-B_{1}\right)$.

Notation 3.18. $[a, b]=a b a^{-1} b^{-1},<a, b>=a b a b^{-1} a^{-1} b^{-1}, a_{b}=b^{-1} a b$.

Proposition 3.19. $G_{1} \doteq \pi_{1}\left(\mathbb{C}^{2}-B_{1}\right)$ is generated by $\left\{j, j^{\prime}\right\}_{j=1}^{8}$ and has the following relations:

(1) $1=1^{\prime}$;

(2) $\langle\underline{6}, \underline{7}\rangle=\langle\underline{4}, \underline{8}\rangle=\langle\underline{1}, \underline{2}\rangle=\langle\underline{3}, \underline{4}\rangle=\langle\underline{5}, \underline{6}\rangle=\langle\underline{7}, \underline{8}\rangle=e$;

(3) $7^{\prime}=6^{-1} 6^{\prime-1} 76^{\prime} 6,4=8^{\prime} 84^{\prime} 8^{-1} 8^{\prime-1}, 1=2^{\prime} 21^{\prime} 2^{-1} 2^{\prime-1}, 4^{\prime}=3^{-1} 3^{\prime-1} 43^{\prime} 3$, $5=6^{\prime} 65^{\prime} 6^{-1} 6^{\prime-1}, 7=8^{\prime} 87^{\prime} 8^{-1} 8^{-1}$

(4) $[\underline{1}, \underline{3}]=[\underline{2}, \underline{4}]=[\underline{1}, \underline{4}]=[\underline{1}, \underline{5}]=[\underline{4}, \underline{5}]=e,[\underline{i}, \underline{6}]=e, 1 \leq i \leq 4$, $[\underline{i}, \underline{7}]=e, 1 \leq i \leq 5, \quad[\underline{i}, \underline{8}]=e, 1 \leq i \leq 6, i \neq 4$;

(5) $\langle\underline{2}, 3\rangle=\left\langle\underline{5}, 3^{\prime}\right\rangle=e, 5^{\prime} 53^{\prime} 5^{-1} 5^{\prime-1}=32^{\prime} 232^{-1} 2^{\prime-1} 3^{-1},\left[3 \underline{2} 3^{-1}, \underline{5}\right]=e$.

Proof. In the proof, we use the Van-Kampen theorem (Theorem 3.6), the complex conjugation method and the invariance relations. Relation (1) is induced from the braid $Z_{11^{\prime}}$. Relations (2) and (3) are induced, using Van-Kampen and invariance, from the factors $H_{i}, 3 \leq i \leq 8$. Relations (4) are induced from the parasitic intersection points - the factors $C_{i}$. Relations (5) are induced from the factors in $\mathrm{H}_{2}$.

Proposition 3.20. The following relations hold in $G_{1}$ :

(6) $\langle\underline{2}, \underline{3}\rangle=\langle\underline{3}, \underline{5}\rangle=\langle\underline{2}, \underline{5}\rangle=e$

(7) $\left[\underline{2}^{-1} \underline{32}, \underline{5}\right]=e$

Proof. By Proposition 3.19 ((5) and (3)), it is known that

$$
e=\left\langle 3^{\prime}, \underline{5}\right\rangle=\left\langle 4^{-1} 34^{\prime} 3^{-1} 4, \underline{5}\right\rangle \underset{[4, \underline{5}]=e}{\overline{=}}\left\langle 34^{\prime} 3^{-1}, \underline{5}\right\rangle \underset{\left\langle 3,4^{\prime}\right\rangle=e}{=}\left\langle 4^{\prime-1} 34^{\prime}, \underline{5}\right\rangle{ }_{\left[4^{\prime}, \underline{5}\right]=e}\langle 3, \underline{5}\rangle .
$$

Thus $\langle\underline{3}, \underline{5}\rangle=e$. Also, we have:

$$
e=\langle\underline{2}, 3\rangle=\left\langle\underline{2}, 4^{\prime} 43^{\prime} 4^{-1} 4^{\prime-1}\right\rangle_{[\underline{4}, \underline{2}]=e}\left\langle\underline{2}, 3^{\prime}\right\rangle \Rightarrow\langle\underline{2}, \underline{3}\rangle=e .
$$

From relation (5) we get $3^{\prime}=5^{-1} 5^{-1} 32^{\prime} 232^{-1} 2^{\prime-1} 3^{-1} 5^{\prime} 5$ and also

$$
\begin{aligned}
& e=\left\langle 3^{\prime}, 5\right\rangle=\left\langle 5^{-1} 5^{\prime-1} 32^{\prime} 232^{-1} 2^{\prime-1} 3^{-1} 5^{\prime} 5,5\right\rangle=\left\langle 32^{\prime} 232^{-1} 2^{\prime-1} 3^{-1}, 5^{\prime} 55^{-1}\right\rangle \\
& \text { Invariance } z_{55^{\prime}}\left\langle 32^{\prime} 232^{-1} 2^{-1} 3^{-1}, 5^{\prime}\right\rangle_{\langle 2,3\rangle=e}=e^{\left\langle 32^{\prime} 3^{-1} 232^{-1} 3^{-1}, 5^{\prime}\right\rangle_{\left[3 \underline{2}^{-1}, 5^{\prime}\right]=e}}\left\langle 2,5^{\prime}\right\rangle
\end{aligned}
$$


and by invariance relations we get $\langle\underline{2}, \underline{5}\rangle=e$. This completes the proof of (6). From (5) we have

$$
e=\left[3 \underline{2}^{-1}, \underline{5}\right]=\left[\underline{2}^{-1} 3 \underline{2}, \underline{5}\right]=\left[\underline{2}^{-1} 4^{\prime} 43^{\prime} 4^{-1} 4^{\prime-1} \underline{2}, \underline{5}\right]_{[\underline{4}, \underline{2}]=[\underline{4}, \underline{5}]=e}\left[\underline{2}^{-1} \underline{3}^{\prime} \underline{2}, \underline{5}\right] .
$$

Thus $\left[\underline{2}^{-1} \underline{32}, \underline{5}\right]=e$.

Our next task is to express the generators $j^{\prime}(j=1,2,3,5, . ., 8)$ by the generators $1 \leq j \leq 8$ and $4^{\prime}$. This is easy: using (3), we get

$$
\text { (8) } \begin{aligned}
& 1^{\prime}=1, \quad 2^{\prime}=1^{-1} 212^{-1} 1, \quad 3^{\prime}=4^{-1} 34^{\prime} 3^{-1} 4, \quad 8^{\prime}=4^{-1} 84^{\prime} 8^{-1} 4 \\
& 7^{\prime}=8^{-1} 8^{\prime-1} 78^{\prime} 8, \quad 6^{\prime}=7^{-1} 67^{\prime} 6^{-1} 7, \quad 5^{\prime}=6^{-1} 6^{\prime-1} 56^{\prime} 6 .
\end{aligned}
$$

Therefore, the group $G$ is generated by the generators $\{j\}_{j=1}^{8} \cup\left\{4^{\prime}\right\}$. We note that all the commutator and triple relations (i.e., (2), (4), (6), (7)) that involve the generators $j^{\prime}$ where $j=1,2,3,5, \ldots, 8$ can be reduced, since these $j^{\prime}$ 's are expressed in terms of the other generators. Our task now is to reduce most of the relations coming from the branch points, i.e. (3) and the second relation at (5). Notice that all of the relations in (3) are already reduced, as we have used them to define the generators $j^{\prime}$ (by (8)). However, one can see that, for example, in the second relation in (5) we can substitute the generators $j^{\prime}$ using (8), till we get an expression containing only the generators $\{j\}_{j=1}^{8} \cup\left\{4^{\prime}\right\}$. Therefore, we get the following relation:

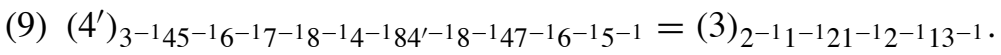

Notation 3.21. Denote relation (9) by $\rho_{1}$.

Note that (9) can be described as a "global" relation, involving almost all the generators of the group. We need only to find out what are the "local" relations, involving only the generators $4,4^{\prime}, 3$ and 8 .

Proposition 3.22. The following relations hold in $G_{1}$ :

(10) $\left\langle 84^{\prime} 8^{-1}, 4\right\rangle=\left\langle 34^{\prime} 3^{-1}, 4\right\rangle=e$.

(11) $\left[3^{-1} 43,84^{\prime} 8^{-1}\right]=e$.

Proof. Knowing that $4^{\prime}=3^{-1} 3^{\prime-1} 43^{\prime} 3$ we see that:

$$
\left\langle 34^{\prime} 3^{-1}, 4\right\rangle=\left\langle 33^{-1} 3^{-1} 43^{\prime} 33^{-1}, 4\right\rangle=\left\langle 3^{\prime}, 4\right\rangle \underset{\text { rel.(2) }}{=} e .
$$

The same is dome for the second relation, using $4^{\prime}=8^{-1} 8^{\prime-1} 48^{\prime} 8$. This proves relation set (10). 
For relation (11), we use the relation $4=8^{\prime} 84^{\prime} 8^{-1} 8^{\prime-1}$.

$$
\begin{aligned}
{\left[3^{-1} 43,84^{\prime} 8^{-1}\right] } & =\left[3^{-1} 8^{\prime} 84^{\prime} 8^{-1} 8^{\prime-1} 3,84^{\prime} 8^{-1}\right]_{[3, \underline{8}]=e}=\left[8^{\prime} 83^{-1} 4^{\prime} 38^{-1} 8^{\prime-1}, 84^{\prime} 8^{-1}\right] \\
& =\left[8^{-1} 8^{\prime} 83^{-1} 4^{\prime} 38^{-1} 8^{\prime-1} 8,4^{\prime}\right] \underset{\text { Inv. } Z_{8,8^{\prime}}}{=}\left[8^{\prime} 3^{-1} 4^{\prime} 38^{\prime-1}, 4^{\prime}\right] \\
& =\left[3^{-1} 4^{\prime} 3,8^{\prime-1} 4^{\prime} 8^{\prime}\right] \underset{\langle 3, \underline{4}\rangle=\left\langle 8^{\prime}, \underline{4}\right\rangle=e}{=}\left[4^{\prime} 34^{\prime-1}, 4^{\prime} 8^{\prime} 4^{\prime-1}\right] \\
& =\left[3,8^{\prime}\right]=e .
\end{aligned}
$$

The last relation we want to induce concerns the fact that once the we have two "circles" in the graph associated to the generators (see Figure 3.6 in Proposition 3.25 ), we ought to find a triple relation relating each two edges that intersect in one vertex.

Proposition 3.23. The following relation holds in $G_{1}$ :

(12) $\left\langle 3^{-1} 43,56784^{\prime} 8^{-1} 7^{-1} 6^{-1} 5^{-1}\right\rangle=e$.

Proof. First, we prove that $\left\langle 3^{-1} 43,5\right\rangle=e$.

$$
\left\langle 3^{-1} 43,5\right\rangle_{\langle 3,4\rangle=e}\left\langle 434^{-1}, 5\right\rangle_{[5,4]} \overline{\overline{4}}=e(3,5\rangle=e .
$$

Thus

$$
\begin{aligned}
& \left\langle 3^{-1} 43,56784^{\prime} 8^{-1} 7^{-1} 6^{-1} 5^{-1}\right\rangle=\left\langle 5^{-1} \cdot\left(3^{-1} 43\right) \cdot 5,6784^{\prime} 8^{-1} 7^{-1} 6^{-1}\right\rangle \\
& =\left\langle 3^{-1} 43 \cdot 5 \cdot\left(3^{-1} 43\right)^{-1}, 6784^{\prime} 8^{-1} 7^{-1} 6^{-1}\right\rangle \\
& {[6,3]=[7,3]=[6,4]=[7,4]=e^{\left\langle 3^{-1} 43 \cdot\left(7^{-1} 6^{-1} 567\right) \cdot\left(3^{-1} 43\right)^{-1}, 84^{\prime} 8^{-1}\right\rangle}} \\
& \underset{\text { rel. (11) }}{=}\left\langle\left(7^{-1} 6^{-1} 567\right) \cdot, 84^{\prime} 8^{-1}\right\rangle{ }_{\langle 5, \overline{6}\rangle=e}\left\langle 7^{-1} 565^{-1} 7,84^{\prime} 8^{-1}\right\rangle \\
& {[5,7]=[5,8]=\left[5,4^{\prime}\right]=e e_{\langle 6,7\rangle=[6,8]=\left[6,4^{\prime}\right]=e}\left\langle 7,84^{\prime} 8^{-1}\right\rangle=\langle 7,8\rangle=e .}
\end{aligned}
$$

Definition 3.24. Let $T$ be a graph with $n$ vertices. In the spirit of [33] and [4], denote by $\hat{A}(T)$ the following generalized Artin group. This is the group generated by the edges $u \in T$ subject to the following relations:

(i) $u v=v u$ if $u$, $v$ are disjoint.

(ii) $u v u=v u v$ if $u, v$ intersect in one vertex. 
(iii) $\left[u, v w v^{-1}\right]=e$ for $u, v, w \in T$ which meet in only one vertex.

(iv) for $u, v, v^{\prime}, w \in T$ which intersect in the following way:

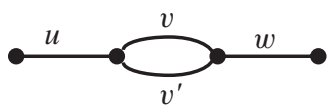

the edges satisfy the relations:

(1) $\left\langle w v^{\prime} w^{-1}, v\right\rangle=\left\langle u v^{\prime} u^{-1}, v\right\rangle=e$

(2) $\left[u^{-1} v u, w v^{\prime} w^{-1}\right]=e$.

(v) For two circles in the graph $T$, embedded in each other in the following way

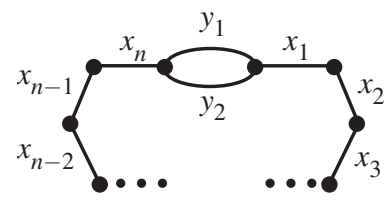

The edges satisfy the relation: $\left\langle x_{n}^{-1} y_{1} x_{n}, x_{n-1} \cdot \ldots \cdot x_{2} x_{1} y_{2} x_{1}^{-1} x_{2}^{-1} \cdot \ldots \cdot x_{n-1}^{-1}\right\rangle=e$.

Summarizing propositions 3.19, 3.20, relation (9), 3.22 and 3.23 we get the following:

Proposition 3.25. $G_{1} \simeq \hat{A}\left(T_{1}\right) / \rho_{1}$, where $T_{1}$ is the following graph:

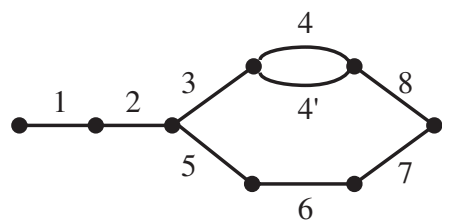

Figure 3.6.

Remark 3.26. Let $T_{1}, T_{2}$ be connected disjoint graphs. Then $\hat{A}\left(T_{1} \cup T_{2}\right)=\hat{A}\left(T_{1}\right) \times$ $\hat{A}\left(T_{2}\right)$. 


\subsection{The fundamental group related to $\mathbb{C P}^{1} \times C_{g}, g>1$}

In this subsection, we compute the BMF of the branch curve $B_{g}$ of $\mathbb{C P}^{1} \times C_{g}, g>1$ and the corresponding fundamental group. We show the connections between these groups and the twisted Artin group defined earlier (see Definition 3.24). We begin with the surface $\mathbb{C P}^{1} \times C_{2}$.

Construction 3.27. As in Construction 3.8, we can build a degeneration of $\mathbb{C P}^{1} \times$ $C_{2}$. Embedding the rational scroll $\mathbb{C P}^{1} \times \mathbb{C P}^{1}$ with respect to the linear system $(1,6)$, we degenerate it into $S^{\prime \prime}$ : a union of 12 planes $S_{i}$. Choosing two pairs of lines $\ell_{1}, \ell_{4}$ in $S_{1}, S_{4}$ and $\ell_{7}, \ell_{10}$ in $S_{7}, S_{10}$, we can attach to each pair a quadric $Q_{j}$, $j=1,7$ such that $Q_{j} \cap S^{\prime \prime}=\ell_{j} \cup \ell_{j+3}$. Degenerating each of the two quadrics into two planes, the union of the 16 planes is a degenerated planar surface which is the degeneration of $\mathbb{C P}^{1} \times C_{2}$, as is proved in [9, Theorem 4.6]. See Figure 3.7 for the degeneration.

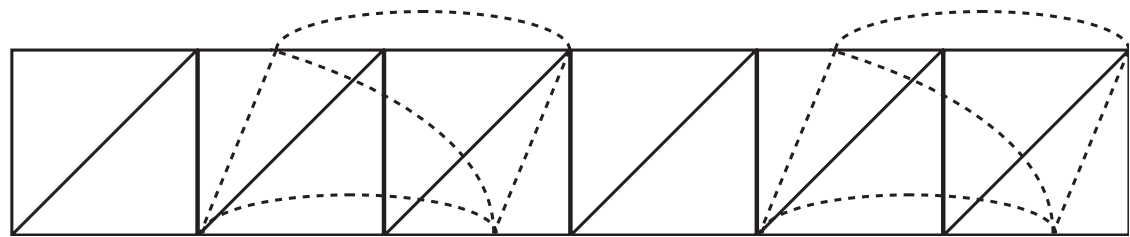

Figure 3.7. Degeneration of $\mathbb{C P}^{1} \times C_{2}$.

Repeating the process described in the previous subsection, we numerate the singularities $v_{i}, 1 \leq i \leq 16$ of the degenerated surface $\mathbb{C P}^{1} \times C_{2}$ and the lines of intersection $L_{i}, 1 \leq i \leq 17$ as follows:

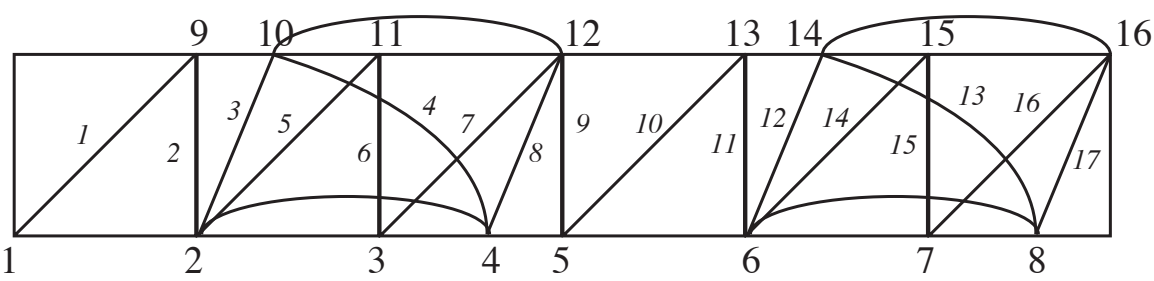

Figure 3.8. Numeration of singular points and lines of the degenerated ramification curve of $\mathbb{C P}^{1} \times C_{2}$.

Once again, we project the degenerated surface to $\mathbb{C P}^{2}$, compute the BMF of the degenerated branch curve $\left(=\Pi_{i=16}^{1} \widetilde{C}_{i} \Delta_{i}^{2}\right)$ and regenerate it.

Remark 3.28. The points $v_{i}, 3 \leq i \leq 16, i \neq 6,12$ are 3-points, and their local regenerated BMFs are either $Z_{a^{\prime}, b b^{\prime}}^{(3)} \cdot \widetilde{Z}_{a a^{\prime}}$ or $Z_{a a^{\prime}, b}^{(3)} \cdot \widetilde{Z}_{b b^{\prime}}$ (where $v_{i}=L_{a} \cap L_{b}$ ). The point $v_{1}$ contributes the factor $Z_{1,1^{\prime}}$ to the global BMF. The local regenerated BMF of a neighborhood of the points $v_{2}, v_{6}, v_{12}$ is computed as in Remark 3.10. 
Theorem 3.29. The braid monodromy factorization of the branch curve $B_{2}$ of a generic projection of $\mathbb{C P}^{1} \times C_{2}$ embedded in $\mathbb{C P} \mathbb{P}^{17}$ is:

$$
\Delta^{2}=\prod_{i=16}^{1} C_{i} \cdot H_{i}
$$

where

$$
\begin{array}{lll}
C_{i}=\mathrm{id}, i=1,9, \ldots, 16, & C_{2}=D_{3} \cdot D_{5}, & C_{3}=D_{6} \cdot D_{7}, \\
C_{4}=D_{4} \cdot D_{8}, & C_{5}=D_{9} \cdot D_{10} & C_{6}=D_{11} \cdot D_{12} \cdot D_{14}, \\
C_{7}=D_{15} \cdot D_{16}, & C_{8}=D_{13} \cdot D_{17} &
\end{array}
$$

where

$$
\begin{aligned}
& D_{3}=\bar{Z}_{11^{\prime}, 33^{\prime}}^{2}, \quad D_{4}=\bar{Z}_{11^{\prime}, 44^{\prime}}^{2} \bar{Z}_{22^{\prime}, 44^{\prime}}^{2}, \quad D_{5}=\Pi_{i=1}^{2} \bar{Z}_{i i^{\prime}, 55^{\prime}}^{2} \cdot Z_{44^{\prime}, 55^{\prime}}^{2}, \\
& D_{6}=\Pi_{i=1}^{4} \bar{Z}_{\substack{i i^{\prime}, 66^{\prime} \\
\left(5-5^{\prime}\right)}}^{2}, \quad D_{7}=\Pi_{i=1}^{5} \bar{Z}_{i i^{\prime}, 77^{\prime}}^{2}, \quad D_{8}=\prod_{i=1}^{3} \bar{Z}_{i i^{\prime}, 88^{\prime}}^{2} \cdot \bar{Z}_{55^{\prime}, 88^{\prime}}^{2} \bar{Z}_{66^{\prime}, 88^{\prime}}^{2} \text {, } \\
& D_{9}=\prod_{i=1}^{6} \bar{Z}_{i i^{\prime}, 99^{\prime}}^{2} \quad D_{10}=\prod_{i=1}^{8} \bar{Z}_{i i^{\prime}, 1010^{\prime}}^{2}, \quad D_{11}=\prod_{i=1}^{9} \bar{Z}_{i i^{\prime}, 1111^{\prime}}^{2}, \\
& D_{12}=\prod_{i=1}^{10} \bar{Z}_{i i^{\prime}, 1212^{\prime}}^{2}, \quad D_{13}=\prod_{i=1}^{11} \bar{Z}_{i i^{\prime}, 1313^{\prime}}^{2}, \quad D_{14}=\prod_{i=1}^{10} \bar{Z}_{i i^{\prime}, 1414^{\prime}}^{2} Z_{1313^{\prime}, 1414^{\prime}}^{2}, \\
& D_{15}=\prod_{i=1}^{13} \bar{Z}_{i i^{\prime}, 1515^{\prime}}^{2}, \quad D_{16}=\prod_{i=1}^{14} \bar{Z}_{i i^{\prime}, 1616^{\prime}}^{2} \\
& D_{17}=\prod_{i=1}^{12} \bar{Z}_{i i^{\prime}, 1717^{\prime}}^{2} \cdot \bar{Z}_{1212^{\prime}, 1717^{\prime}}^{2} \bar{Z}_{1515^{\prime}, 1717^{\prime}}^{2}
\end{aligned}
$$

and

$$
\begin{gathered}
H_{1}=Z_{1,1^{\prime}}, \quad H_{i}=Z_{a^{\prime}, b b^{\prime}}^{(3)} \cdot \widetilde{Z}_{a a^{\prime}} \text { for } i=4,8,9,11,13,15,16, \\
H_{i}=Z_{a a^{\prime}, b}^{(3)} \cdot \widetilde{Z}_{b b^{\prime}} \text { for } i=3,5,7,10,14,
\end{gathered}
$$

$H_{i}=\varphi(a, b, c)$, where $i=2,6,12$ and $v_{i}$ is the intersection of the lines of $L_{a}, L_{b}, L_{c}$, and $L_{b}$ is regenerated first.

Let $G_{2} \doteq \pi_{1}\left(\mathbb{C}^{2}-B_{2}\right)$ be the fundamental group of the complement of the branch curve. 
Proposition 3.30. $G_{2}$ is isomorphic to a quotient of $\hat{A}\left(T_{2}\right)$, where $T_{2}$ is the following graph:

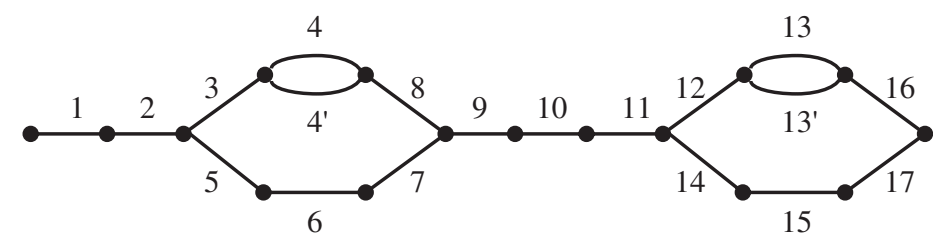

Figure 3.9.

Proof. The existence of the relations (i)-(v) as in Definition 3.24 is induced from the braid monodormy factorization of $B_{2}$, using the Van-Kampen theorem, as in Propositions 3.19, 3.20, 3.22.

Notation 3.31. We introduce the following notations

(i) Let $T$ be a connected planar graph, with no repeated edges, and the valence of each vertex is $\leq 3$. We denote these requirements by $\otimes$.

(ii) For a graph $T=(E, V), v \in V$, denote by $E_{T, v}=E_{v}$ the set of all the edges in $T$ one of whose ends is $v$.

(iii) $E_{v}^{0}=E \backslash E_{v}$.

(iv) Let $\mathrm{T}$ be a graph satisfying $\otimes$. Denote by $R\left(E_{v}\right)$ the following expression, induced from the edges in $E_{v}$ :

(A) $E_{v}=\left\{u_{1}, u_{2}\right\}$, then $R\left(E_{v}\right)=u_{1} u_{2} u_{1} u_{2}^{-1} u_{1}^{-1} u_{2}^{-1}$, where:

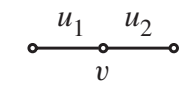

(B) $E_{v}=\left\{u_{1}, u_{2}, u_{3}\right\}$, then $R\left(E_{v}\right)=u_{1} u_{2} u_{3} u_{2}^{-1} u_{1}^{-1} u_{2} u_{3}^{-1} u_{2}^{-1}$, where:

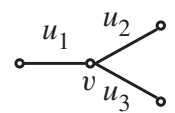

Definition 3.32. Let $T_{1}=\left(V_{1}, E_{1}\right), T_{2}=\left(V_{2}, E_{2}\right)$ be two graphs satisfying $\otimes$. Assume there exist two vertices $v_{1} \in V_{1}, v_{2} \in V_{2}$ such that the degree $d\left(v_{1}\right)=i<$ 3 and $d\left(v_{2}\right) \leq 3-i$. We create a new graph $T_{1} \bigcup_{v_{1}}^{v_{2}} T_{2}$ by identifying the vertices $v_{1}$ and $v_{2}$. Note that $T_{1} \bigcup_{v_{1}}^{v_{2}} T_{2}$ also satisfies $\otimes$. Let $v$ be the identified vertex $v_{1}=v_{2}$ in $T_{1} \bigcup_{v_{1}}^{v_{2}} T_{2}$. For example, see the following figure: 
$T_{1}$

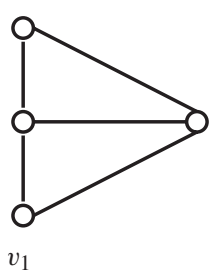

$T_{2}$

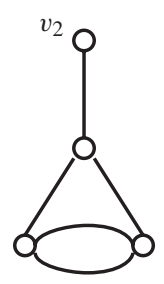

$T_{1} \bigcup_{v_{1}}^{v_{2}} T_{2}$

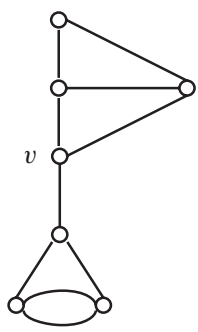

\section{Proposition 3.33.}

$$
\begin{aligned}
& \hat{A}\left(T_{1} \bigcup_{v_{1}}^{v_{2}} T_{2}\right) \\
& =\left\{\hat{A}\left(T_{1}\right) * \hat{A}\left(T_{2}\right) \mid \begin{array}{l}
{\left[u_{1}, u_{2}\right]=e, \quad u_{1} \in E_{v_{1}}^{0}, u_{2} \in E_{2} \text { or } u_{2} \in E_{v_{2}}^{0}, u_{1} \in E_{1}} \\
R\left(E_{v}\right)=e
\end{array}\right\} .
\end{aligned}
$$

Proof. We first note that the degree of $v_{1}$ is less than 3 , so the only possible cases are:

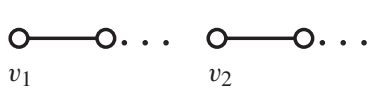

(a)

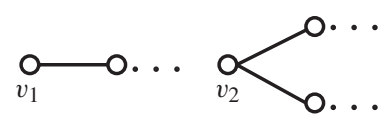

(b)

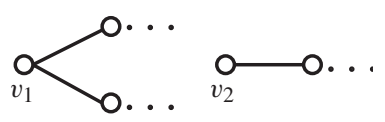

(c)

Cases (b) and (c) are actually the same, so we consider only cases (a) and (b). Since the edges of $T_{1}, T_{2}$ are not changed under the identification of $v_{1}$ and $v_{2}$, it is obvious that the relations in $\hat{A}\left(T_{1}\right)$ and $\hat{A}\left(T_{2}\right)$ are satisfied in $\hat{A}\left(T_{1} \bigcup_{v_{1}}^{v_{2}} T_{2}\right)$. In addition, for an edge $u_{1} \in E_{1}$ such that $u_{1} \notin E_{v_{1}}, u_{1}$ is disjoint from any edge $u_{2} \in$ $E_{2}$. Thus, in $\hat{A}\left(T_{1} \bigcup_{v_{1}}^{v_{2}} T_{2}\right)$, the generator corresponding to $u_{1}$ commutes with any generator corresponding to $u_{2}$. The same is true for an edge $u_{2} \notin E_{v_{2}}$ and edges in $E_{1}$. We only have to take into account the relation induced from the identification of $v_{1}$ and $v_{2}$. Consider case (a). $E_{v}$ is a set of two adjacent edges $u, w$, intersecting at $v$. So in $\hat{A}\left(T_{1} \bigcup_{v_{1}}^{v_{2}} T_{2}\right)$, by Definition (3.24)(ii), we would have the relation $v w v=$ $w v w$, or $R\left(E_{v}\right)=e$. We follow the same arguments for case (b).

\section{Notation 3.34.}

(i) Let $T_{1}=\left(V_{1}, E_{1}\right)$ be the graph in proposition 3.25, $T_{0}=\left(V_{0}, E_{0}\right)$, and let $\delta \in V_{1}, \alpha, \beta \in V_{0}$ be the following vertices:
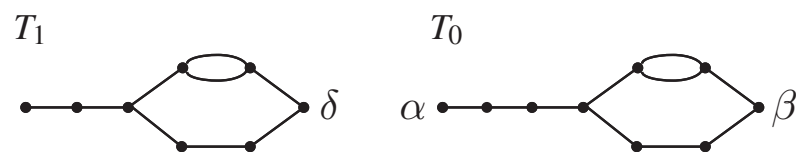
(ii) For $1<g$, take $g-1$ copies of $T_{0}$, and denote by $\alpha_{i}, \beta_{i}, 1 \leq i \leq g$ the corresponding vertices in each $T_{0}$. Let $T_{g} \doteq T_{1} \bigcup_{\delta}^{\alpha_{1}} T_{0} \bigcup_{\beta_{1}}^{\alpha_{2}} \ldots \bigcup_{\beta_{g-1}}^{\alpha_{g}} T_{0}$.

(iii) We now construct a degenerated model of $\mathbb{C P}^{1} \times C_{g}$, where $C_{g}$ is a genus $g$ curve. Embed $\mathbb{C P}^{1} \times \mathbb{C P}^{1}$ by the linear system $(1,3 g)$, degenerate it to a union of $6 g$ planes, attach $g$ quadrics to $g$ pairs of non-intersecting planes and then degenerate the quadrics, as was done in Constructions 3.8 and 3.27. The resulting degeneration should be composed from $g$ "building blocks" as in Figure 3.4. Explicitly
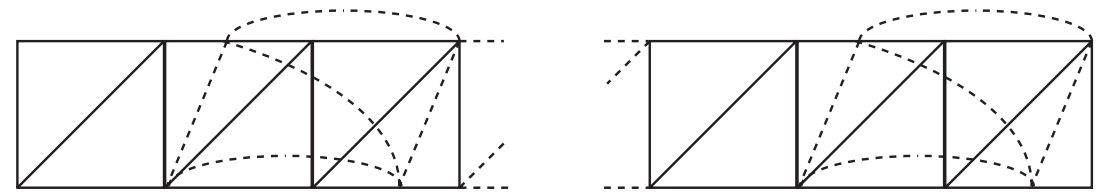

Figure 3.10. Degeneration of $\mathbb{C P}^{1} \times C_{g}$ embedded in $\mathbb{C} \mathbb{P}^{8 g+1}$.

Denote by $S_{g}$ this surface whose degeneration is as in Figure 3.11 above. Consider a generic projection $\mathbb{C P}^{8 g+1} \rightarrow \mathbb{C P}^{2}$ and its restriction to $S_{g}$, we denote by $B_{g}$ the branch curve and $G_{g}=\pi_{1}\left(\mathbb{C}^{2}-B_{g}\right)$ the corresponding fundamental group.

We saw (Proposition 3.25) that $G_{1} \simeq \hat{A}\left(T_{1}\right) / \rho_{1}$ and that $G_{2}$ is a quotient of $\hat{A}\left(T_{2}\right)$. Thus, by induction, we have the following

Theorem 3.35. $G_{g}$ is isomorphic to a quotient of $\hat{A}\left(T_{g}\right)$.

\subsection{The fundamental group of the Galois cover of $\mathbb{C P}^{1} \times C_{g}$}

In this subsection we find the fundamental group of the Galois cover of $\mathbb{C P}^{1} \times C_{g}$, generalizing the results of [1], [2] and using the method outlined in [20]. We start with reviewing the known facts on the fundamental group of the Galois cover of a surface.

Let $S$ be a projective surface of degree $n$. Given a generic projection $\pi$ : $S \rightarrow \mathbb{C P}^{2}$, we define the Galois cover as the closure of the $n$-fold fibered product $S_{\mathrm{Gal}}=\overline{S \times \times_{\pi} \ldots \times_{\pi} S-\Delta}$ where $\Delta$ is the generalized diagonal. We denote by $S_{\mathrm{Gal}}^{\mathrm{aff}}$ the affine part of $S_{\mathrm{Gal}}$.

Let $B$ be the branch curve of $\pi: S \rightarrow \mathbb{C P}^{2}$. It is known that we have the following exact sequences (see e.g., [25]):

$$
\begin{aligned}
& 0 \rightarrow \pi_{1}\left(S_{\mathrm{Gal}}^{\mathrm{aff}}\right) \rightarrow \pi_{1}\left(\mathbb{C}^{2}-B\right) /\left\langle\Gamma^{2}=1\right\rangle \rightarrow \operatorname{Sym}_{n} \rightarrow 0, \\
& 0 \rightarrow \pi_{1}\left(S_{\mathrm{Gal}}\right) \rightarrow \pi_{1}\left(\mathbb{C P}^{2}-B\right) /\left\langle\Gamma^{2}=1\right\rangle \rightarrow \operatorname{Sym}_{n} \rightarrow 0 .
\end{aligned}
$$

Let $\delta=\prod \Gamma_{i}$ the product of all the standard topological generators of $\pi_{1}\left(\mathbb{C}^{2}-B\right)$. Recall that $\pi_{1}\left(\mathbb{C P}^{2}-B\right)=\pi_{1}\left(\mathbb{C}^{2}-B\right) /\langle\delta=1\rangle$. Then, by [20, Proposition 5.10] 
and Theorem 3.35, we see that for the surface $S_{g}$ (see Notation 3.34(iii))

$$
H_{1}\left(\left(S_{g}\right)_{\mathrm{Gal}}\right) \simeq \mathbb{Z}^{2 g(8 g-1)}
$$

We consider $\delta$ as an element in $\pi_{1}\left(S_{\mathrm{Gal}}^{\text {aff }}\right)$. Denote $Z=\langle\delta\rangle \cap \mathbb{Z}$ (see $[20$, Theorem 4.5]). Then we have the following exact sequence (see [20, Proposition 5.10]):

$$
0 \rightarrow \mathbb{Z} / Z \rightarrow \pi_{1}\left(\left(S_{g}\right)_{\mathrm{Gal}}\right) \rightarrow \mathbb{Z}^{2 g(8 g-1)} \rightarrow 0
$$

In order to compute $\pi_{1}\left(\left(S_{g}\right)_{\text {Gal }}\right)$ we need the following definition.

Definition 3.36. The generalized Coxeter group $\hat{C}(T)$ associated to a graph $T$ is defined as

$$
\hat{C}(T)=\hat{A}(T) /\left\langle\Gamma^{2}=1\right\rangle
$$

where $\Gamma$ goes over all the generators of $\hat{A}(T)$.

Note that $\pi_{1}\left(\left(S_{g}\right)_{\mathrm{Gal}}\right)$ is a subgroup of a quotient of $\hat{C}\left(T_{g}\right)$, by Theorem 3.35 and the short exact sequence (3.1).

\section{Theorem 3.37.}

$$
\pi_{1}\left(\left(S_{g}\right)_{\mathrm{Gal}}\right) \simeq \mathbb{Z}^{2 g(8 g-1)}
$$

Proof. We will prove the theorem only for $g=1$, where for the $g>1$ the proof is similar. Let us consider the following group

$$
H=G_{1} /\left\langle\Gamma^{2}=1, \Gamma_{4}=\Gamma_{4^{\prime}}\right\rangle=\hat{C}\left(T_{1}\right) /\left\langle\Gamma_{4}=\Gamma_{4^{\prime}}\right\rangle .
$$

Examining the relations in $G_{1}$, we see that the relation $\rho_{1}$ becomes trivial under the new added relations (see Notation 3.21 and Proposition 3.25). Therefore, the group $H$ is in fact isomorphic to the following Coxeter group $H \simeq C_{Y}(T)$ (see [33] for the definition of the Coxeter group $\left.C_{Y}(T)\right)$, where $T$ is as in the figure below.

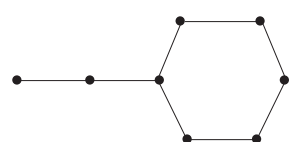

Figure 3.11. The graph $T$ associated to the Coxeter group $H$.

Therefore, by [33, Theorem 6.1], $H \simeq S y m_{8} \ltimes A_{1,8}=S y m_{8} \ltimes \mathbb{Z}^{7}$ (see [33] for the notation of $\left.A_{t, n}\right)$. As this group is infinite, its associated Coxeter element $\prod \Gamma_{i}$ has infinite order (see e.g. [16, page 175]). Thus its order is infinite also in the group $G_{1} /\left\langle\Gamma^{2}=1\right\rangle$ and thus in any subgroup of it, for example in $\pi_{1}\left(\left(S_{g}\right)_{\mathrm{Gal}}^{\mathrm{aff}}\right)$. Therefore, the order of $\delta \in \pi_{1}\left(S_{\mathrm{Gal}}^{\mathrm{aff}}\right)$ is infinite, and $Z=\langle\delta\rangle \cap \mathbb{Z}=\mathbb{Z}$. Considering the exact sequence in (3.3), we are done. 


\section{References}

[1] M. Amram, D. Goldberg, M. Teicher and U. Vishne, The fundamental group of a Galois cover of $\mathbb{C P}^{1} \times \mathbb{T}$, Algebr. Geom. Topol. 2 (2002), 403-432.

[2] M. AmRam and D. GoldberG,Higher degree Galois covers of $\mathbb{C P}^{1} \times \mathbb{T}$, Algebr. Geom. Topol. 4 (2004), 841-859.

[3] M. AmRAM, M. TEICHER and U. Vishne, The Coxeter quotient of the fundamental group of a Galois cover of $\mathbb{T} \times \mathbb{T}$, Comm. Algebra 34 (2006), 89-106.

[4] M. Amram, R. Shwartz and M. Teicher, Coxeter covers of the classical Coxeter groups, Int. J. Algebra Comput 20 (2010), 1041-1062.

[5] M. Amram and O. Shoetsu, Degenerations and fundamental groups related to some special Toric varieties, Michigan Math. J. 54 (2006), 587-610.

[6] M. AmRAM, M. FriedmAn and M. TEICHER, The fundamental group of the complement of the branch curve of the second Hirzebruch surface, Topology 48 (2009), 23-40.

[7] M. AmRAM, M. FRIEDMAN and M. TEICHER, The fundamental group of the complement of the branch curve of $\mathbb{C P}^{1} \times \mathbb{T}$, Acta Mathematica Sin. (Engl. Ser.) 25 (2009), 1443-1458.

[8] D. Auroux, S. K. Donaldson, L. Katzarkov and M. Yotov, Fundamental groups of complements of plane curves and symplectic invariants, Topology 43 (2004), 1285-1318.

[9] A. Calabri, C. Ciliberto, F. Flamini and R. Miranda, Degenerations of scrolls to unions of planes, Atti Accad. Naz. Licei, Cl. Sci. Fis. Mat. Natur. Rend. Lincei (9) Mat. Appl. 17 (2006), 95-123.

[10] A. Calabri, C. Ciliberto, F. Flamini and R. Miranda, On the $K^{2}$ of degenerations of surfaces and the multiple point formula, Ann. of Math. 165 (2007), 335-395.

[11] A. Calabri, C. Ciliberto, F. Flamini and R. Miranda, On degenerations of surfaces, arXiv:math/0310009v2 [math.AG] (2008).

[12] M. Friedman and M. TeICHER, The regeneration of a 5-point, Pure Appl. Math. Q. 4 (2008) (Fedor Bogomolov special issue, part I), 383-425.

[13] M. FRIEDMAN and M. TEICHER, On non fundamental group equivalent surfaces, Algebr. Geom. Topol. 8 (2008) 397-433.

[14] M. FRIEDMAN and M. TEICHER, On fundamental groups related to the Hirzebruch surface $F_{1}$, Sci. China Ser. A. 51 (2008), 1-18.

[15] R. Hartshorne, "Families of Curves in $\mathbb{C P}^{3}$ and Zeuthen's Problem", Mem. Amer. Math. Soc. 130,1997 , no. 617.

[16] J. E. Humphreys, "Reflection Groups and Coxeter Groups", Cambridge University Press, 1992.

[17] ViK. Kulikov, On Chisini's conjecture, Izv. Ross. Akad. Nauk Ser. Mat. 63 (1999), 83116.

[18] ViK. Kulikov, On Chisini's conjecture II, Izv. Math. 72 (2008).

[19] V. S. KULIKOV and VIK.S. KULIKOV, On complete degenerations of surfaces with ordinary singularities in $\mathbb{P}^{3}$, Mat. Sb. 201 (2010), 129-160.

[20] C. LiEDTKE, On fundamental groups of Galois closures of generic projections, Trans. Amer. Math. Soc. 362 (1010), 2167-2188. arXiv:0505.406.

[21] B. Moishezon, Algebraic surfaces and the arithmetic of braids. II, In: "Combinatorial Methods in Topology and Algebraic Geometry", Rochester, NY, (1982), Contemp. Math., Vol. 44, Amer. Math. Soc., Providence, RI, (1985) 311-344.

[22] B. Moishezon, On cuspidal branch curves, J. Algebraic Geom. 2 (1993), 309-384.

[23] B. Moishezon, A. RoBB and M. TeICHER, On Galois covers of Hirzebruch surfaces, Math. Ann. 305 (1996), 493-539.

[24] B. MoISHEZON and M. TeICHER, Galois covers in theory of algebraic surfaces, In: "Algebraic Geometry Bowdoin, 1985”, Proc. Sympos. Pure Math., Vol. 46, Part 2, Amer. Math. Soc., Providence, RI, 1987, pp. 47-65. 
[25] B. MoISHEZON and M. TEICHER, Simply connected algebraic surfaces of positive index, Invent. Math. 89 (1987), 601-643.

[26] B. MoISHEZON and M. TeICHER, Braid group technique in complex geometry, I: Line arrangements in $\mathbb{C P}^{2}$, Contemp. Math. 78 (1988), 425-555.

[27] B. MOISHEZON and M. TEICHER, Braid group technique in complex geometry, II: From arrangements of lines and conics to cuspidal curves, In: "Algebraic Geometry", Lecture Notes in Math., Vol. 1479, 1990, 131-180.

[28] B. MOISHEZON and M. TEICHER, Braid group techniques in complex geometry III: Projective degeneration of $V_{3}$, Contemp. Math. 162 (1994), 313-332.

[29] B. MoISHEZON and M. TEICHER, Braid group techniques in complex geometry IV: Braid monodromy of the branch curve $S_{3}$ of $V_{3} \rightarrow \mathbb{C P}^{2}$ and application to $\pi_{1}:\left(\mathbb{C P}^{2}-S_{3}, *\right)$, Contemp. Math. 162 (1993), 332-358.

[30] B. MoISHEZON and M. TEICHER, Braid group technique in complex geometry V: The fundamental group of a complement of a branch curve of a Veronese generic projection, Comm. Anal. Geom. 4 (1996), 1-120.

[31] A. Rовв, "The Topology of Branch Curves of Complete Intersections", Doctoral Thesis, Columbia University, 1994.

[32] A. RoBB, On branch curves of algebraic surfaces, In: "Singularities and Complex Geometry" (Bijug, 1994), AMS/IP Stud. Adv. Math. 5, Amer. Math. Soc., Providence, RI, 1997, pp. 193-221.

[33] L. Rowen, M. TeICHER and U. Vishne, Coxeter covers of the symmetric groups, J. Group Theory 8 (2005), 139-169.

[34] M. TEICHER, New invariants for surfaces, Contemp. Mathematics 231 (1999), 271-281.

[35] M. TEICHER, On the quotient of the braid group by commutators of transversal half-twists and its group actions, Topology Appl. 78 (1997), 153-186.

[36] G. ZAPPA, Sulla degenerazione delle superficie algebriche in sistemi di piani distinti, con applicazioni allo studio delle rigate, Atti R. Accad. d'Italia, Mem. Cl. Sci. FF., MM. e NN. 13 (1943), 989-1021.

[37] O. ZARISKI, On the topological discriminant group of a Riemann surface of genus $p$, Amer. J. Math. 59 (1937), 335-358.

Department of Mathematics

Bar-Ilan University

52900 Ramat Gan, Israel

fridmam@macs.biu.ac.il

teicher@macs.biu.ac.il 